\title{
REVIEWS
}

Check for updates

\section{Phenotypic diversity and metabolic specialization of renal endothelial cells}

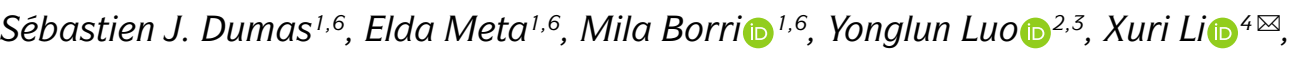 \\ Ton J. Rabelink ${ }^{5}$ and Peter Carmeliet (1) $^{1,4 凶}$
}

Abstract | Complex multicellular life in mammals relies on functional cooperation of different organs for the survival of the whole organism. The kidneys play a critical part in this process through the maintenance of fluid volume and composition homeostasis, which enables other organs to fulfil their tasks. The renal endothelium exhibits phenotypic and molecular traits that distinguish it from endothelia of other organs. Moreover, the adult kidney vasculature comprises diverse populations of mostly quiescent, but not metabolically inactive, endothelial cells (ECs) that reside within the kidney glomeruli, cortex and medulla. Each of these populations supports specific functions, for example, in the filtration of blood plasma, the reabsorption and secretion of water and solutes, and the concentration of urine. Transcriptional profiling of these diverse EC populations suggests they have adapted to local microenvironmental conditions (hypoxia, shear stress, hyperosmolarity), enabling them to support kidney functions. Exposure of ECs to microenvironment-derived angiogenic factors affects their metabolism, and sustains kidney development and homeostasis, whereas EC-derived angiocrine factors preserve distinct microenvironment niches. In the context of kidney disease, renal ECs show alteration in their metabolism and phenotype in response to pathological changes in the local microenvironment, further promoting kidney dysfunction. Understanding the diversity and specialization of kidney ECs could provide new avenues for the treatment of kidney diseases and kidney regeneration.

The mammalian vascular system consists of two connected and highly branched networks that pervade the whole body - each with specific roles. The blood vascular system delivers oxygen and nutrients to parenchymal tissues, and facilitates waste removal, immune surveillance and immune cell trafficking, coagulation, and the production of angiocrine signals for tissue maintenance and regeneration ${ }^{1}$. By contrast, the lymphatic vascular system drains the extravasated interstitial fluid from permeable blood capillaries back to the veins, and facilitates immune cell trafficking and lipid transport ${ }^{2}$. All blood vessels are lined with blood endothelial cells (BECs, referred to as ECs hereafter), whereas lymphatic endothelial cells (LECs) form the innermost layer of lymphatic vessels - with each population supporting its vasculature-specific tasks. However, endothelial heterogeneity extends far beyond the broad differences between blood and lymphatic endothelium. In particular, ECs from different organs exhibit unique molecular profiles that support the specific functions of the $\operatorname{organ}^{3-6}$. The kidney benefits from a highly specialized vasculature, which is tightly linked to the kidney epithelial system ${ }^{7}$. Specifically, phenotypically distinct populations of renal endothelial cells (RECs) coexist within the three anatomical and functional compartments of the kidney, the glomeruli, cortex and medulla - where they support specific kidney tasks ${ }^{8,9}$. Importantly, technological advances have enabled the study of REC heterogeneity at the single-cell level, providing new insights into their specialized roles in kidney health and disease $\mathrm{f}^{6,10,11}$.

The kidney is critical for the maintenance of organismal homeostasis, regulating the volume and composition of body fluids ${ }^{7}$. Kidneys receive $20-25 \%$ of cardiac output and exhibit a stereotypic blood vessel architecture. This architecture not only enables the delivery of oxygen and nutrients to the kidneys, but also enables participation in the filtration of blood plasma, the reabsorption of ions and metabolites from the filtrate, the secretion of ions and metabolites in the primary urine, and urine concentration ${ }^{7,8}$. These highly orchestrated processes enable fine-tuning of extracellular fluid volume, blood pressure, osmolality and ion concentration $^{7,8}$. The kidneys also regulate circulating metabolite levels, not only by excreting metabolic waste, but also by releasing glucose (via gluconeogenesis) and amino acids, for example ${ }^{12}$. Mammalian organs continually exchange metabolites via the circulation, with selective usage to support their own metabolic activities ${ }^{12}$. 


\section{Key points}

- The endothelium differs between different organs, probably to support distinct organ functions.

- Multiple specialized endothelial cell phenotypes coexist within the renal glomeruli, cortex and medulla; these function to support glomerular filtration, the reabsorption and secretion of ions and metabolites, and urine concentration.

- The different local microenvironments in the kidney shape the molecular and metabolic heterogeneity of the renal endothelium; conversely, endothelial cell-derived angiocrine factors sustain the niches of different kidney microenvironments.

- The metabolism of renal endothelial cells can be altered in the context of kidney injury and diseases, partly as a result of changes in the microenvironment.

- Greater understanding of the phenotypic diversity and metabolic specialization of renal endothelial cells may aid identification of new targets for the treatment of kidney diseases and kidney regeneration.

Accordingly, the metabolic functions of ECs within different organs probably demonstrate organ-specific differences, as supported by findings from metabolic transcriptome analyses of ECs in different organs ${ }^{6}$. Furthermore, the metabolic plasticity of ECs allows them to adapt and respond to environmental changes with respect to their metabolic needs and functions ${ }^{3,13}$. Emerging evidence suggests that the specialized RECs of the kidney tailor their metabolic transcriptome to support kidney function ${ }^{10}$.

REC dysfunction accompanies the acute or progressive loss of kidney function ${ }^{14,15}$. This dysfunction is associated with an increase in arterial vasoconstriction and a reduction in renal blood flow, the acquisition of pro-inflammatory and pro-thrombotic phenotypes that favour immune cell adhesion and infiltration and the formation of microthrombi, the dissociation of mural pericytes from the endothelial layer, breakdown of the endothelial barrier resulting in interstitial oedema, rarefaction of peritubular capillaries (thereby promoting kidney hypoxia), and endothelial-to-mesenchymal transition, which contributes to kidney fibrosis ${ }^{16,17}$, suggesting that the endothelium could be targeted to protect against kidney injury and/or to regenerate kidney function.

This Review summarizes our current understanding of the kidney vasculature, focusing on recent advances in our understanding of the phenotypic, molecular and metabolic heterogeneity of RECs in relation to their microenvironment. We also discuss the potential application of targeting REC metabolism as a therapeutic strategy in kidney diseases or for kidney regeneration.

\footnotetext{
Author addresses

'Laboratory of Angiogenesis and Vascular Metabolism, Department of Oncology, KU Leuven and Center for Cancer Biology, VIB, Leuven, Belgium.

'Lars Bolund Institute of Regenerative Medicine, Qingdao-Europe Advanced Institute for Life Sciences, BGI-Qingdao, BGI-Shenzhen, Qingdao, China.

${ }^{3}$ Department of Biomedicine, Aarhus University, Aarhus, Denmark.

${ }^{4}$ State Key Laboratory of Ophthalmology, Zhongshan Ophthalmic Center, Sun Yat-Sen University, Guangzhou, China.

${ }^{5}$ The Einthoven Laboratory for Vascular and Regenerative Medicine, Department of Internal Medicine, Division of Nephrology, Leiden University Medical Center, Leiden, Netherlands.

${ }^{6}$ These authors contributed equally: Sébastien J. Dumas, Elda Meta and Mila Borri.
}

\section{Renal endothelium heterogeneity Renal vascular anatomy}

The kidney is supplied with blood via the renal artery, which after entering the kidney via the renal hilum, branches into segmental, interlobar, arcuate and interlobular arteries (FIG. 1a), and ultimately, afferent arterioles, which are high-resistance vessels responsible for the control of the glomerular blood flow and glomerular filtration rate $(\mathrm{GFR})^{18}$. From the afferent arterioles the blood enters the glomerular tuft - a network of highly fenestrated glomerular capillaries where ultrafiltration of blood plasma occurs at a rate of $\sim 120-140 \mathrm{ml} / \mathrm{min}$ in adult humans, enabling low molecular weight solutes to pass from the glomerular capillaries to the Bowman's space ${ }^{19}$. After a fraction of the plasma has been filtered, blood leaves the glomerular tuft through efferent arterioles to vascularize the distal and proximal convoluted tubules, forming the cortical peritubular capillary network. Blood within the peritubular capillaries is enriched with high molecular weight solutes and has a low fluid content owing to the loss of fluid during glomerular ultrafiltration. Thus, peritubular capillaries are dedicated to the reabsorption of water, ions and essential nutrients from the proximal ${ }^{7,20}$ and distal tubules ${ }^{21}$. Several ions such as $\mathrm{H}^{+}\left(\mathrm{REF}^{22}\right)$ and $\mathrm{K}^{+}$ $\left(\right.$ REF. $\left.^{23}\right)$ as well as molecules such as creatinine ${ }^{24}$ and drug metabolites ${ }^{25}$ - which were not completely filtered by the glomerular capillaries but still need to be eliminated from the body - move from the peritubular capillaries into the epithelial cells of the proximal ${ }^{20}$ or distal tubules to be secreted and eliminated in the urine ${ }^{21}$. The efferent arterioles from the juxtamedullary nephrons give rise to the descending vasa recta (DVR), which interconnects with the ascending vasa recta (AVR) through capillary plexuses. The AVR and DVR run countercurrent to the loop of Henle, and participate in medullary countercurrent exchange, which as described later, is necessary to maintain an osmolarity gradient for urine concentration ${ }^{26}$. Eventually, the cortical and medullary capillary systems together with the AVR coalesce into a venous system at the corticomedullary junction. More specifically, the renal venous vasculature drains the blood from the peritubular capillaries and AVR into the interlobular and arcuate veins and then interlobar veins, ultimately forming the renal vein that emerges from the kidney hilum and finally branches into the inferior vena cava ${ }^{7}$.

The kidney is also supplied by lymphatic vessels, which follow the overall topography of the kidney blood vasculature ${ }^{27}$ (FIG. 1a). They are mainly present in the kidney cortex where their primary role is to remove fluid and macromolecules (such as albumin) from the interstitial space between the tubules and capillaries $^{28}$. They also have a role in the infiltration of immune cells and subsequent inflammation ${ }^{29}$. In the glomeruli, they surround the Bowman's capsule without penetrating into the glomerular tuft ${ }^{28}$. By contrast, traditional lymphatic vessels are rarely present in the kidney medulla; in this region, interstitial fluid and macromolecules are removed by the AVR, which represents a type of hybrid blood vessel with lymphatic-like features $^{28,30}$. 

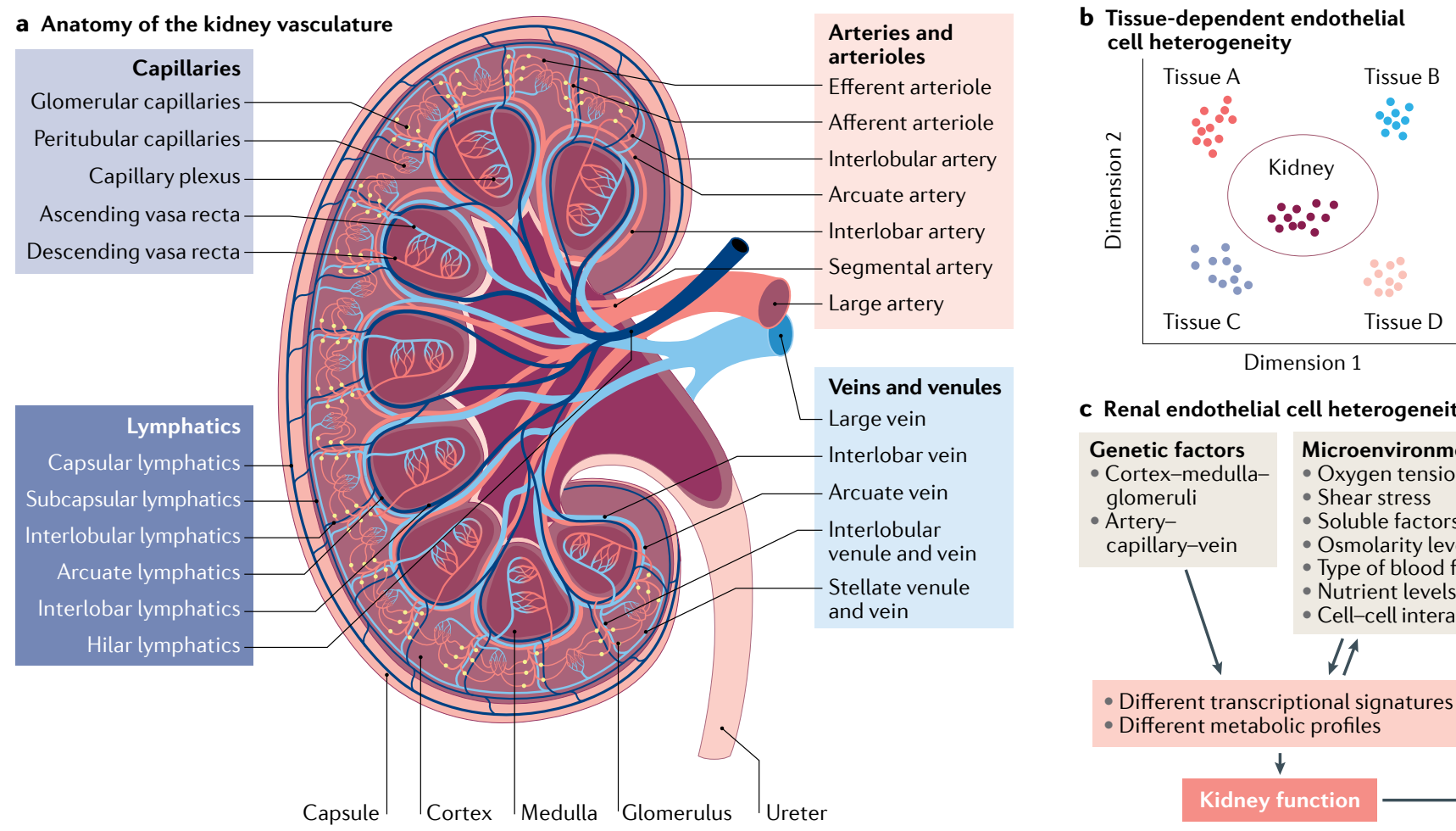

c Renal endothelial cell heterogeneity

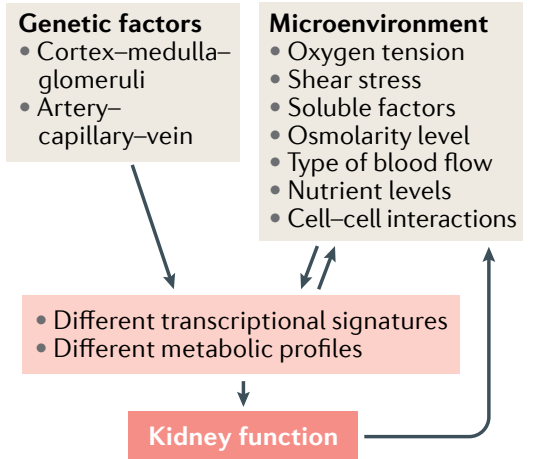

Fig. 1 | Anatomy and heterogeneity of the kidney vasculature. a The human kidney contains a highly specialized vasculature that is closely linked to the kidney epithelial system. The blood vascular system delivers oxygen and nutrients to parenchymal tissues, and facilitates waste removal, immune surveillance and immune cell trafficking, coagulation, and the production of angiocrine signals for tissue maintenance and regeneration, whereas the lymphatic vascular system drains the extravasated interstitial fluid from permeable blood capillaries back to the veins, and facilitates immune cell trafficking and lipid transport. $\mathbf{b} \mid$ Endothelial cells (ECs) are characterized by their tissue-specific transcriptional heterogeneity. The plot shows a representative dimensionality reduction of the transcriptome profiles of ECs isolated from kidney and four other hypothetical tissues or organs. $\mathrm{c} \mid \mathrm{EC}$ heterogeneity within the kidney is dictated by a combination of genetic factors and exposure to different microenvironments, which ultimately contribute to kidney function.

\section{Renal endothelial cell phenotypes}

ECs from different organs are phenotypically heterogeneous $^{3-6,31}$. The unique properties of RECs, and in particular glomerular ECs, have long been appreciated. Global transcriptional profiling of ECs from mice has confirmed the existence of organ-specific transcriptome signatures $^{3,4,6}$. Of note, these studies have demonstrated that RECs are the most dissimilar to ECs from other organs including the brain, heart, lung, muscle and testis $^{4}$ (FIG. 1 b) through their expression of genes associated with interferon signalling, as well as genes that encode the angiocrine factors FGF1 and IL-33 (REFS' ${ }^{4,6}$ ). The organ-specific heterogeneity of ECs probably underlies their molecular adaptation to fulfil specific functional roles s $^{3-6,31}$.

However, the heterogeneity of RECs extends beyond the organotypic level, with remarkable diversity of the kidney vasculature, as demonstrated initially by electron microscopy and microarray studies and subsequently by single-cell analyses ${ }^{6,32,33}$. The kidney cortex, glomeruli and medulla contain unique EC populations (cRECs, gRECs and mRECs, respectively). This diversity in EC populations might arise from exposure to the different microenvironments of these regions. For example, the glomerular endothelium is exposed to a high vascular pressure and interacts tightly with podocytes to regulate ultrafiltration, whereas mRECs are exposed to high osmolarity and hypoxia, which are related to the maintenance of an osmolarity gradient and urine concentration $^{6,9,10,32}$ (FIG. 1c).

Beyond inter-compartmental heterogeneity, RECs also demonstrate intra-compartmental heterogeneity, which is probably determined by a number of genetic and environmental factors, including the type of vascular bed (arterial, capillary, venous), their interactions with other cell types (for example, smooth muscle cells, pericytes, granular cells, podocytes and tubule epithelial cells) and their exposure to different microenvironments within the same compartment, such as exposure to different types of flow or different levels of osmolarity ${ }^{34}$ (FIG. 1 1). Developments in single-cell transcriptomics technologies have enabled the heterogeneity of mouse RECs to be mapped at very high resolution ${ }^{6,10,11}$, revealing up to 24 transcriptionally different REC populations ${ }^{6,10,11}$. Of note, the findings from single-cell RNA-seq studies summarized below remain to be confirmed at the protein level, both to comprehensively verify the spatial localization of the predicted proteins and also to integrate knowledge of the post-translational changes and/or signalling mechanisms that may affect protein activity. Also of note is the fact that the relative enrichment of a gene within a particular REC population as determined 
a Glomerular endothelial cell heterogeneity

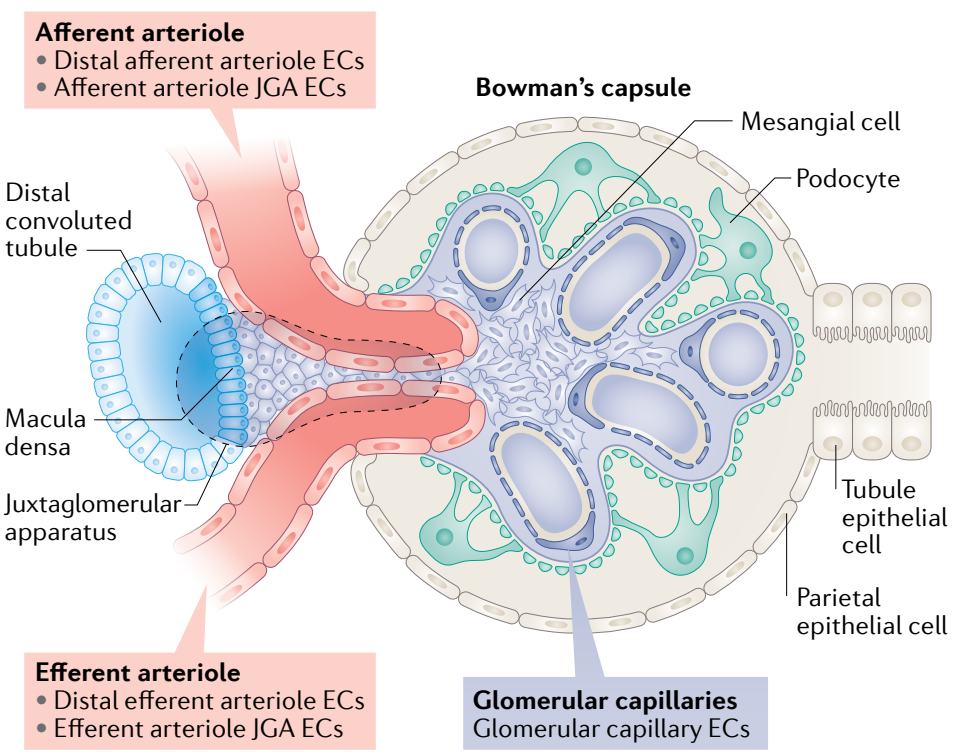

b Glomerular filtration barrier

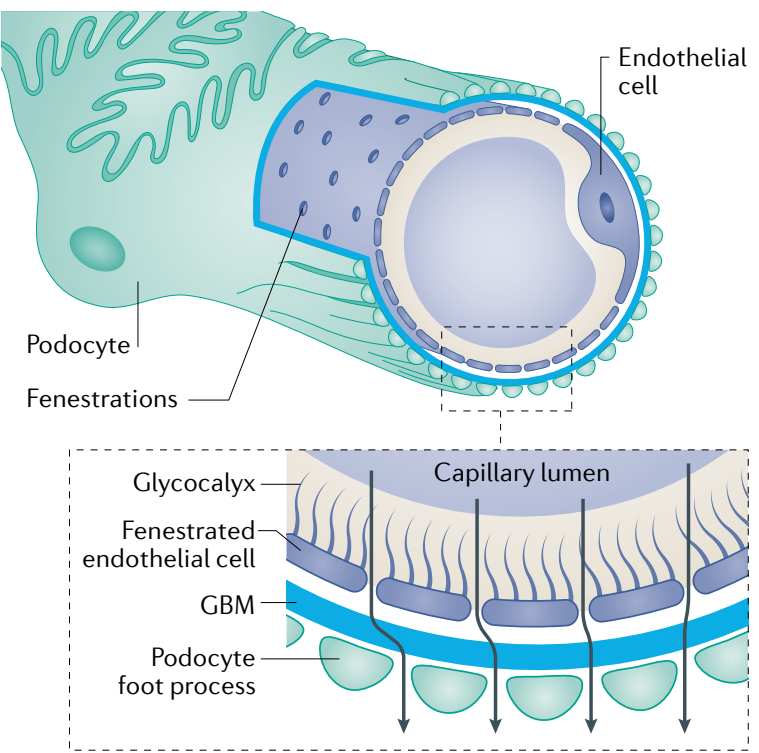

c Glomerular renal endothelial cells: marker genes

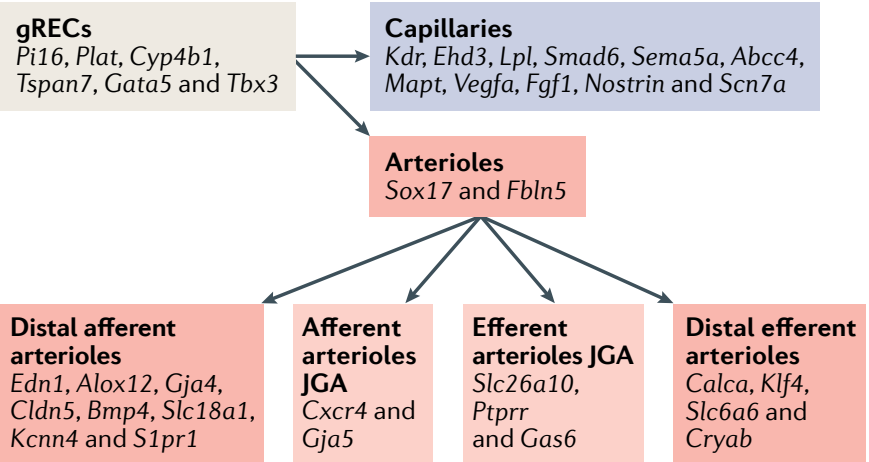

d Heterogeneity of the afferent arteriole

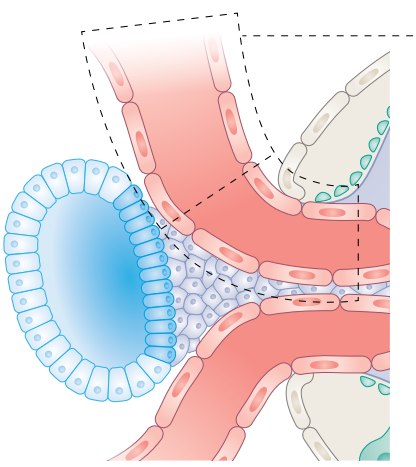

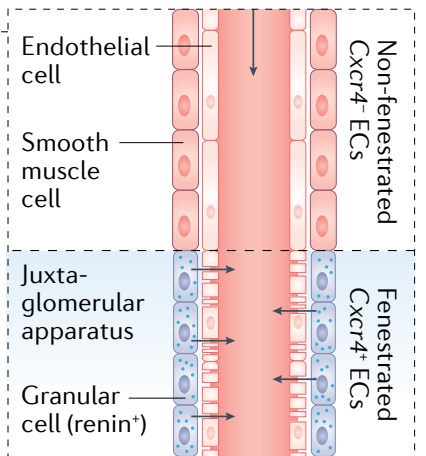

Fig. 2 | Phenotypic and molecular heterogeneity of the glomerular endothelium. a | The glomerular vasculature comprises the glomerular capillaries connected to the afferent and efferent arterioles. The arterioles are in partial contact with the juxtaglomerular apparatus (JGA), which comprises the macula densa of the distal convoluted tubule, granular renin-producing cells and extraglomerular mesangial cells. $\mathbf{b} \mid$ The glomerular filtration barrier is composed of glomerular capillary endothelial cells (ECs) and podocytes, separated by a glomerular basement membrane (GBM). The glomerular capillary endothelium encompasses non-diaphragmed fenestrations and a thick glycocalyx, which sustain glomerular filtration and permselectivity. $\mathbf{c}$ | The heterogeneity of glomerular renal ECs (gRECs) is demonstrated by the differential expression of genes by different gREC types. Pink and purple reflect arteriole and capillary EC phenotypes, respectively. Since REC subpopulations express a combination of several markers, these are indicated following a hierarchical system. More detailed information can be found in Supplementary Table 1. d | The layer of vascular smooth muscle cells from the afferent arteriole is progressively replaced by renin-expressing granular cells within the JGA at the entrance of the glomerular tuft. A switch from a non-fenestrated and $C x c r 4$-negative endothelium to a fenestrated and Cxcr4-positive endothelium within the JGA is also observed.

by single-cell sequencing does not necessarily imply that the expression of that gene is restricted to that specific cell population.

Heterogeneity of glomerular renal endothelial cells. The kidney glomerulus is a highly specialized structure that is responsible for the filtration of blood plasma to generate a primary urine filtrate while ensuring that essential plasma proteins are retained in the blood. It is composed of glomerular capillaries that lie between afferent and efferent arterioles, which are resistance vessels that control both capillary blood flow and pressure. The arterioles of a nephron are in partial contact with the juxtaglomerular apparatus (JGA) - a specialized structure that comprises the macula densa of the distal convoluted tubule, granular renin-producing cells that are associated with the afferent arteriole and extraglomerular mesangial cells (FIG. 2a). The JGA regulates single-nephron GFR and blood pressure through the tubuloglomerular feedback, the myogenic response and the release of renin ${ }^{19,35-37}$.

The glomerular capillary endothelium is composed of unique ECs with non-diaphragmed fenestrations that allow the filtration of high volumes of fluid ${ }^{38}$ (FIG. 2 b). 
The fenestrations are 50-100 $\mathrm{nm}$ in size and occupy around $20 \%$ of the cell surface area, appearing on electron microscopic imaging as trans-cellular holes ${ }^{38}$. The diameter of these fenestrations is theoretically large enough to allow the passage of fluid and large proteins into the tubules. However, capillary gRECs also produce a thick layer of glycocalyx comprising negatively charged glycoproteins and polysaccharides that act as a barrier to protein passage ${ }^{39,40}$. Moreover, plasma components are adsorbed in the glycocalyx and form a broader coat called the endothelial surface layer ${ }^{41}$, which with its filamentous structure further enhances the permselectivity of the glomerular endothelial barrier ${ }^{42}$. Indeed, albuminuria and proteinuria are observed upon glycocalyx impairment ${ }^{39,43,44}$. Together with podocytes, capillary gRECs also synthesize and share a common extracellular matrix known as the glomerular basement membrane (GBM), which comprises mainly collagen type IV, laminin and sulfated proteoglycans ${ }^{42}$. Mutations affecting the synthesis of any of the components of the GBM lead to proteinuria ${ }^{45,46}$. Thus, capillary gRECs, podocytes and the GBM form an efficient glomerular filtration barrier. Of note, the glomerular capillary endothelium lacks a diaphragm and therefore does not express the type II transmembrane glycoprotein plasmalemma vesicle-associated protein 1 (PV1), which is encoded by Plvap ${ }^{10}$ and is a typical marker of fenestrated ECs associated with the bridging diaphragms of endothelial fenestrae and caveolae ${ }^{47}$.

The development and maintenance of the capillary gREC fenestrations require podocyte-derived vascular endothelial growth factor (VEGF), which acts in a paracrine manner through endothelial VEGF receptor 2 (VEGFR2, also known as KDR) ${ }^{48}$. Overexpression of VEGF causes glomerular collapse, rapid loss of capillary gRECs and massive proteinuria ${ }^{49}$. Thus, tight regulation of podocyte VEGF is needed to establish the glomerular vasculature during embryonic development, and for the maintenance of fenestrations in mature glomerular capillaries $^{38,42}$. Similarly, tubular epithelial cell-derived VEGF allows maintenance of the peritubular capillary network ${ }^{50}$.

Unlike other capillary ECs, gRECs are exposed to a high blood pressure and high blood flow, which drives the glomerular filtration process and exposes gRECs to substantial shear stress ${ }^{51}$. Accordingly, gRECs express high levels of the shear stress-regulated transcript Pi16 $\left(\right.$ REFS $^{6,10,11,52}$ ) (FIG. 2c; Supplementary Table 1). Capillary gRECs also express a number of other markers ${ }^{10,11,32,53}$, including Ehd3, which encodes a member of the EHD protein family ${ }^{10,11,38,54,55}$ that regulates endocytic recycling and is thought to regulate the recycling of VEGFR2 in capillary gRECs (FIG. 2C), together with EHD4 (REF. ${ }^{54}$ ). Thus, EHD3 might contribute to the maintenance of glomerular capillary fenestrations. Capillary gRECs also show enriched expression of genes associated with the TGF $\beta$-BMP signalling pathway (Eng, Smad6, Smad7, Xiap and Hipk2 (REFS $\left.{ }^{10,56-58}\right)$ ), which is involved in glomerular capillary formation. Overexpression of TGF $\beta$ induces proteinuria and glomerulosclerosis ${ }^{59}$, and thus the presence of inhibitory SMADs, such as those encoded by Smad6 and Smad7 in gRECs may prevent excessive TGF $\beta$ signalling and glomerular dysfunction. By contrast, podocyte-derived BMP is crucial for normal glomerular capillary formation ${ }^{60}$. Capillary gRECs also specifically express Nostrin ${ }^{32}$, the protein product of which binds endothelial nitric oxide synthase (eNOS) to trigger its translocation from the plasma membrane to vesicle-like subcellular structures, and attenuates the production of nitric oxide (NO) - an important regulator of $\mathrm{GFR}^{32}$. Moreover, the restricted expression of lipoprotein lipase $(L p l)$ to capillary gRECs suggests that the glomerular endothelium may be essential for the release of fatty acids into the ultrafiltrate, which could subsequently be used as an energy source by tubule epithelial cells or for the regulation of blood lipid content, or could contribute to the accumulation of glomerular lipids as observed in pathological contexts ${ }^{6,10,61,62}$. Interestingly, renal expression of genes involved in lipid metabolism correlates with GFR and inflammation in patients with diabetic kidney disease, whereas defective fatty acid oxidation (FAO) in tubular cells contributes to the development of kidney fibrosis ${ }^{61,62}$.

Transcription factors, such as SOX17 and COUP-TFII (encoded by $\mathrm{Nr} 2 \mathrm{f2}$ ) in arterial and venous RECs, respectively, drive transcriptomic signatures and the identity of specific vascular beds $s^{63,64}$. The identity of gRECs relies on the activity of at least two transcription factors: GATA5 and TBX3 (REFS ${ }^{10,11,32}$ ) (FIG. 2C), which mediate the acquisition of a gREC-like gene expression profile when overexpressed together in human umbilical vein ECs (HUVECs), a commonly used EC model ${ }^{11}$. The GATA5 regulon is upregulated in gRECs but not in other REC populations ${ }^{10,11}$ and selective deletion of Gata5 in ECs causes glomerular lesions ${ }^{65}$. Moreover, EC-specific deletion of $T b x 3$ causes morphogenic defects such as microaneurysms in subsets of glomeruli, reduced numbers of capillary gREC fenestrations and deformed podocyte foot processes, suggesting a role for this transcription factor in maintaining the structural organization of glomerular capillaries ${ }^{11}$. In addition, both GATA5 and TBX3 are involved in the regulation of blood pressure. GATA5 affects typical vascular function, protein kinase A and NO signalling pathways ${ }^{65}$, whereas TBX3 is thought to modulate blood pressure via the regulation of renin secretion in the kidney ${ }^{11}$.

Regulation of the vascular tone of afferent and efferent arterioles is required to maintain the constantly high glomerular capillary pressure needed for glomerular filtration ${ }^{18}$. This regulatory process enables a constant GFR to be maintained despite changes in systemic pressure and cardiac output ${ }^{66}$. Afferent arterioles have one to three layers of vascular smooth muscle cells (VSMCs), which, in proximity to the JGA, are partially replaced by renin-producing granular cells ${ }^{67}$ (FIG. 2d). EC heterogeneity also exists within the afferent arteriole, with non-diaphragmed fenestrations of the endothelium nearest to the $\mathrm{JGA}^{68,69}$ - similar to that of the glomerular capillary endothelium - probably to facilitate the rapid transport of renin into the blood ${ }^{18}$ (FIG. 2d). Expression of Gja5 (encoding connexin 40), is enriched in this subset of gRECs ${ }^{10,70}$, and has an important role in communication between the endothelium and granular cells in the JGA to regulate renin release ${ }^{35,70,71}$. These ECs are also 
enriched in other genes involved in cell-to-cell interaction, such as those related to the Wnt and Notch signalling pathways, Ephrin and cytokines and chemokines (FIG. 2C), which might mediate crosstalk between mesangial cells and/or granular cells and gRECs in the JGA, and potentially contribute to autoregulation and blood pressure modulation ${ }^{10}$.

By contrast, gRECs in the upstream (most distal) part of the afferent arterioles express genes involved in vasotone regulation such as $E d n 1$ (which encodes endothelin 1), Alox12 (arachidonate 12-lipoxygenase) and S1pr1 (sphingosine-1-phosphate receptor 1$)^{10,72,73}$ (FIG. 2C). The S1P-S1PR1 signalling pathway potently regulates afferent arteriole vasotone by activating the eNOS system ${ }^{74-76}$. In line with this role, the $\mathrm{S} 1 \mathrm{P}$ receptor is enriched in gRECs in the afferent arterioles and is not detected in efferent arterioles ${ }^{10}$.

In contrast to gRECs in the afferent arterioles, gRECs in the efferent arterioles show lower connexin expression $^{77}$, especially connexin 37 and connexin 40 (encoded by Gja4 and Gja5, respectively) ${ }^{10}$. Similar to ECs from the afferent arterioles, however, transcriptome analyses of ECs from the efferent arterioles indicate the presence of two gREC populations: one presumably associated with the JGA (expressing genes associated with immune cell adhesion and extravasation, and EC permeability) and a second that corresponds to the distal portion of the efferent arteriole (enriched in genes involved in hyperosmolarity responses) ${ }^{10}$ (FIG. 2C).

These insights suggest that the phenotypic and functional diversity of gRECs underlies the ability of these endothelia to maintain GFR through the active modulation of glomerular blood flow and by ensuring glomerular filtration efficiency. Through the integration of tubuloglomerular feedback and myogenic signals, gRECs associated with the JGA in particular are probably critical regulators of GFR.

Heterogeneity of cortical renal endothelial cells. In addition to the glomerular capillary endothelium and the pre-glomerular and post-glomerular afferent and efferent arterioles, the kidney cortex contains lymphatic vessels, and large arteries and veins together with their associated vasa vasora, postcapillary venules and peritubular capillaries. In line with their role in the reabsorption and secretion of solutes, ions and water, cortical peritubular capillaries are thin-walled capillaries comprising ECs that are functionally coupled to the tubular epithelium $^{9}$ (FIG. 3a). Compared with gRECs and mRECs, cRECs - in particular peritubular capillary ECs express high levels of $I g f b p 3$ (encoding insulin-like growth factor-binding protein 3), and Npr3 (encoding natriuretic peptide receptor 3$)^{10,11}$ (FIG. 3b).

The cortical peritubular capillaries arise from the efferent arterioles and surround the proximal and distal convoluted tubules (FIG. 3a), providing oxygen and nutrients, and contributing to the uptake of solutes and water reabsorption from the tubular lumen ${ }^{9}$. Unlike glomerular capillaries, peritubular capillary ECs express Plvap, the protein product of which (PV1) spans the peritubular capillary EC fenestrae. These diaphragmed fenestrae are $62-68 \mathrm{~nm}$ in diameter and probably facilitate the exchange of water, ions and small solutes with proximal and distal tubules ${ }^{9,10}$.

Glomeruli filter approximately $180 \mathrm{~g}$ of glucose per $\mathrm{day}^{78}$ and under physiological conditions, almost all of it is reabsorbed in the proximal tubules. Filtered glucose is first reabsorbed from the lumen of the proximal tubules inside the epithelial cells through sodium-glucose cotransporters (SGLTs). Once the intracellular glucose concentration exceeds that of the interstitium, it diffuses into the interstitial space through specific facilitated glucose transporters (GLUTs), from where it is reabsorbed into the bloodstream ${ }^{79}$. In line with their role in this process, peritubular capillary ECs express higher levels of Slc2a1 (encoding GLUT1) than ECs of other renal vascular beds ${ }^{11}$ (FIG. 3b), suggesting that glucose reabsorption might be facilitated by GLUT1 in peritubular capillary ECs.

Cortical peritubular capillaries include two EC populations - one that expresses high levels of Apoe (encoding apolipoprotein E) and one that expresses little or no Apoe ${ }^{10}$ (FIG. 3b). The Apoe-high population shows enriched expression of other genes related to lipid metabolism such as $P l p p 3$ and Thrs $p^{10,80,81}$. By contrast, the Apoe-low population expresses genes encoding VEGF receptors (Kdr, Flt1 and Nrp1, encoding VEGFR2, VEGFR1 and neuropilin 1, respectively), insulin-like growth factor-binding proteins and receptor ( $I g f b p 5$, Igfbp3 and Insr), and Npr3, which encodes a receptor for natriuretic peptide, which regulates blood volume and sodium excretion ${ }^{10,82-85}$. Whether these two EC populations exist in separate capillaries that interact with proximal convoluted tubules or distal tubules, or whether they exist in the same capillaries is currently unknown.

Surprisingly, two additional capillary EC populations have also been described in the mouse renal cortex - an angiogenic-like EC population and a population that is characterized by the expression of interferon-stimulated genes and genes involved in antigen processing and presentation ${ }^{10}$ (FIG. 3b). The angiogenic-like ECs might have a role in the regeneration of damaged RECs, whereas the interferon-activated ECs might participate in immune surveillance, although further studies are needed to investigate these possibilities ${ }^{10}$.

\section{Fig. 3 | Phenotypic and molecular heterogeneity} of the cortical and medullary renal endothelium. a Phenotypically distinct renal endothelial cell (REC) phenotypes coexist within the two main anatomical compartments of the kidney, the cortex and medulla. b | Markers of different cortical REC (cREC) phenotypes. Since REC subpopulations express a combination of several markers, these are indicated following a hierarchical system. c| Markers of different medullary REC (mREC) populations. Since REC subpopulations express a combination of several markers, these are indicated following a hierarchical system. More detailed information regarding the expression and function of genes expressed in cortical and medullary RECs can be found in Supplementary Table 1. d|Phenotypic differences exist between the descending vasa recta (DVR) and the ascending vasa recta (AVR). The arterial-like ECs of the DVR are non-fenestrated and covered by a pericyte layer that regulates the medullary blood flow. By contrast, the venous-like ECs of the AVR are highly fenestrated and lack pericyte coverage, which facilitates water reuptake. 
cRECs in large arteries are characterized by the expression of the arterial transcription factor gene Sox 17 and the tight junction gene Cldn5 (claudin 5), whereas
cRECs in large veins are characterized by the expression of the transcription factor Nr2f2 (COUP-TFII), and the fenestration marker Plvap ${ }^{6,10,11,47,63,64,86}$ (FIG. 3b).

\section{a Cortical and medullary renal endothelial cell heterogeneity}

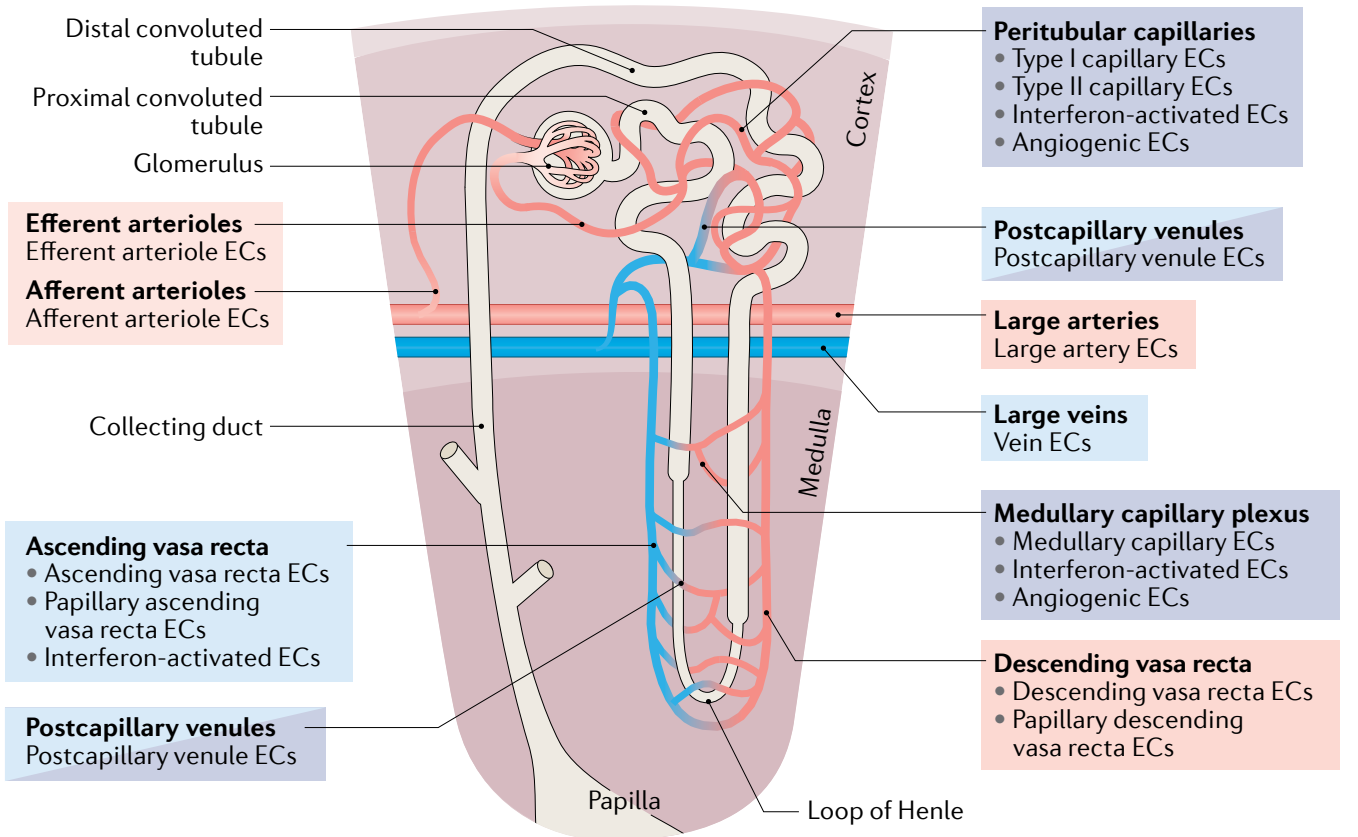

b Cortical renal endothelial cells: marker genes

\begin{tabular}{|c|c|c|c|c|c|}
\hline \multirow{3}{*}{$\begin{array}{l}\text { Cortical } \\
\text { endothelium } \\
\text { cRECs } \\
\text { Igfbp3 } \\
\text { and Npr3 }\end{array}$} & \multirow[b]{2}{*}{$\begin{array}{l}\text { Arteries } \\
\text { Sox17, Sema3g and Fbln5 } \\
\text { Veins } \\
\text { Nr2f2, CD9, Gas6 and Plvap }\end{array}$} & \multirow{2}{*}{\multicolumn{2}{|c|}{$\begin{array}{l}\text { Large arteries } \\
\text { Eln, Ltbp4, Bmp4, Mgp, } \\
\text { Ace, Edn1, Gja5, S1pr1, } \\
\text { Slc8a1, Cldn5, Jag1 } \\
\text { and Kcnn4 }\end{array}$}} & \multirow[b]{2}{*}{$\begin{array}{l}\text { Afferent arterioles } \\
\text { Edn1, Alox12, Gja4, } \\
\text { Cldn5, Bmp4, } \\
\text { Slc18a1, Kcnn4, } \\
\text { S1pr1 and Cxcl12 }\end{array}$} & \multirow[b]{2}{*}{$\begin{array}{l}\text { Efferent } \\
\text { arterioles } \\
\text { Calca, Klf4, } \\
\text { Slc6a6 and } \\
\text { Cryab }\end{array}$} \\
\hline & & & & & \\
\hline & $\begin{array}{l}\text { Postcapillary venules } \\
K d r, N r 2 f 2, T n \times b \text { and Jup }\end{array}$ & $\begin{array}{l}\text { Type I } \\
\text { capillaries } \\
\text { High Apoe. }\end{array}$ & $\begin{array}{l}\text { Type II } \\
\text { capillaries } \\
\text { Low }\end{array}$ & $\begin{array}{l}\text { Interferon } \\
\text { activated } \\
\text { Isa15. Ifit1. }\end{array}$ & $\begin{array}{l}\text { Angiogenic } \\
\text { Gpihbp1, Esm1, }\end{array}$ \\
\hline $\begin{array}{l}\text { Lymphatics } \\
\text { Lyve1, Prox1, } \\
\text { Flt4 and Pdpn }\end{array}$ & $\begin{array}{l}\text { Peritubular capillaries } \\
\text { Kdr, Flt1, Nrp1,Plvap, } \\
\text { Insr and Slc2a1 }\end{array}$ & $\begin{array}{l}\text { Plpp3 and } \\
\text { Thrsp }\end{array}$ & Apoe & $\begin{array}{l}\text { Ifit3, Ifi203, } \\
\text { Ifit } 3 \text {, Ifit2, Irf7 } \\
\text { and Ifi204 }\end{array}$ & $\begin{array}{l}\text { Aplnr, Apln, } \\
\text { Trp53i11, Plk2 } \\
\text { and Fscn1 }\end{array}$ \\
\hline
\end{tabular}

C Medullary renal endothelial cells: marker genes

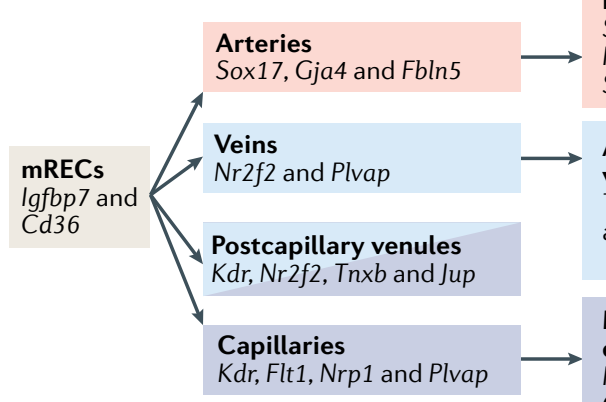

Descending vasa recta Slc14a1, Aqp1, Scin, Cxcl12,

Hpgd, Edn1, Adipor2, Cldn5, Slc5a3 and Crip1

\section{Ascending Ascending vasa recta vasa recta papilla \\ Tek, Gas6 Cryab, Fxyd2, Car2, Cd9, S100a6, Akr1b3, Fxyd5, Nrgn, Ifi203, Ifit3b, Ifit2, Irf7 Aldoa, Crip1, Gapdh and Ldha and Ifi204 \\ Ascending vasa recta interferon activated interferon activate}

Medullary Interferon activated Angiogenic capillaries Isg15, Ifit1, Ifit3,

Plpp3 and Ifi203, Ifit3b, Ifit2, Irf7 Cd36
Descending vasa recta papilla

S100a4, S100a6, Akr1b3, Fxyd5 and Nrgn Gpihbp1, Esm1, Col4a1, Col4a2, Aplnr, Apln, Trp53i11, Plk2 and Fscn1 d

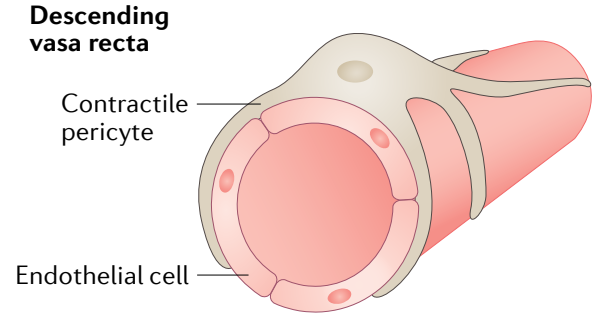

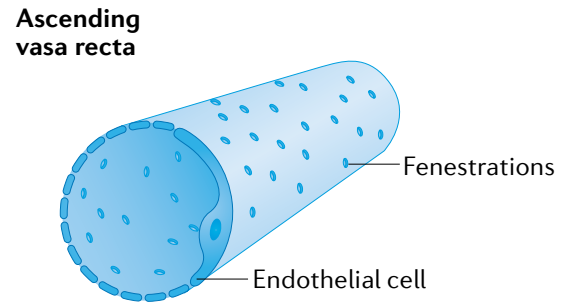




\section{Tunica media}

The middle layer of the arterial and vein walls, localized between the tunica intima, formed by the endothelial cell layer, and the tunica adventitia, which mostly includes fibroblasts and collagens. The tunica media of arteries comprise vascular smooth muscle cells with or without elastic fibres. In veins, the tunica media consists of connective tissue and elastic fibres and sometimes includes one layer of smooth muscle cells.
Arterial cRECs express the semaphorin-encoding gene Sema3g, which has autocrine and paracrine effects on ECs and VSMCs, respectively, the connexin-encoding genes Gja4 and Gja5, which are components of myoendothelial junctions, and the Notch family member Jag1 (REFS ${ }^{6,10,11,87-90}$ ) (FIG. 3b). Large arteries are exposed to high blood pressure and their vascular tone is modulated in response to changes in blood pressure. Their ability to respond to mechanical signals is enabled by the presence of an elastic layer in the tunica media that is rich in elastic fibres ${ }^{9,91}$, and through the expression of genes related to elastic fibre assembly such as Eln (elastin), $L t b p 4$ (latent-transforming growth factor- $\beta$-binding protein 4 ), Fbln5 (fibulin 5) and Bmp4 (REFS ${ }^{6,10,11,92-95}$ ). They also express high levels of $M g p$ (matrix Gla protein) ${ }^{6,10}$, which suppresses vascular calcification probably through inhibition of BMP2 and BMP4 signalling ${ }^{96}$. Consistent with their role in regulating renal blood flow, cRECs of the large arteries also express genes that are responsible for vasotone regulation such as Ace, $E d n 1$ and S1pr1 (REFS ${ }^{6,10,97-99}$ ) (FIG. 3b).

The delivery of oxygen and nutrients and the removal of waste products released within the vascular wall of large arteries and veins are facilitated by the vasa vasora ${ }^{100}$. Vasa vasora RECs were not identified in the published studies of mouse single-cell RECs, presumably because vessels with a lumen diameter of $<0.5 \mathrm{~mm}$ (the diameter of normal vessels in mice) do not normally have vasa vasora ${ }^{6,10,11,100}$. Performing such studies in larger animals or in humans, which have larger renal vessels, may increase the likelihood of capturing vasa vasora RECs. There are currently no markers described for RECs derived from vasa vasora.

Beyond the blood vascular system, the renal cortex also contains two sets of renal lymphatic vessels. Both of these originate as blind-ended capillaries in the renal lobule from where one set follows the arteries towards the hilum to connect the hilar and capsular system and the other penetrates the capsule to join the capsular lymphatics $^{28,101}$ (FIG. 1a). The renal lymphatic capillaries can be distinguished from blood vessel capillaries as they are mainly present in the interstitium, are blind-ended and lack pericytes ${ }^{28,29}$. Renal lymphatic capillaries consist of single-layered, 'oak-leaf'-shaped, partially overlapping $\mathrm{LECs}^{28,29}$, which can be distinguished from BECs by the expression of several markers ${ }^{6}$, of which the best-known are $P d p n$ (podoplanin) ${ }^{102}$, the hyaluronan receptor-encoding gene Lyve1 (REF. ${ }^{103}$ ), Flt4 (which encodes VEGFR3) ${ }^{104}$ and the transcription factor gene Prox1 (REF. ${ }^{105}$ ) (FIG. 3b). Although these markers are also expressed in other cell types, they can be used to distinguish between the two major EC types ${ }^{29}$. In the human kidney, podoplanin has been described as being the most reliable marker of LECs ${ }^{28,29}$. Nonetheless, neither of the two known kidney LEC populations have been identified in published single-cell RNA-seq studies ${ }^{6,10,11}$, possibly owing to their loss during technical processing steps (for example, during enzymatic digestion or EC purification), and/or because they represent too small an EC fraction compared with the population of renal BECs. Further studies are therefore needed to characterize the heterogeneity of the renal lymphatic endothelium.
Heterogeneity of the medullary renal endothelial cells. The primary role of the renal medulla is urine concentration ${ }^{9}$. The anatomical arrangement of the vasa recta and low blood flow of the renal medulla $(10 \%$ of total renal blood flow ${ }^{9}$ ), prevent wash-out of solutes, such as urea and $\mathrm{NaCl}$, creating an osmolarity gradient from the outer medulla to the renal papilla, which is essential for urine concentration ${ }^{26,106}$. This gradient varies according to hydration status ${ }^{106}$.

The renal medullary endothelium is characterized by the expression of $I g f b p 7\left(\right.$ REFS $\left.^{10,11}\right)$, a urinary marker of kidney injury that predicts renal recovery after acute kidney injury (AKI) ${ }^{107}$, and $C d 36\left(\mathrm{REFS}^{10,32}\right.$ ), which encodes a scavenger receptor that is responsible for the uptake of long-chain fatty acids from the circulation ${ }^{108}$ (FIC. 3c). Hence, lipids might shuttle in a CD36-dependent manner through the medullary endothelium to medullary interstitial cells, a fibroblast-like cell population that is characterized by lipid droplets, the abundance of which correlates with the state of diuresis ${ }^{109}$. Deletion of $C d 36$ in mice was associated with an increased kidney-dependent risk of spontaneous hypertension ${ }^{10,110}$, but attenuated the development of kidney fibrosis in response to a high-fat $\operatorname{diet}^{111}$ (FIG. 3c), therefore suggesting both a protective and a pathological role for lipid transport in these processes.

Like the cortical and glomerular endothelia, the renal medullary endothelium exhibits extensive intra-compartmental heterogeneity ${ }^{10,11}$. The DVR are arterial-like vessels comprising a continuous endothelium surrounded by smooth muscle-like pericytes or VSMCs that respond to vasoactive stimuli to control renal medullary blood flow. Consistent with their arteriolar-like phenotype, DVR ECs express Sox17 (REFS ${ }^{10,55}$ ), Cldn5 (REFS ${ }^{10,55,86,112}$ ), Fbln5, Gja4 and Cxcl12 (CXCL12, also known as SDF1 - a chemokine protein that acts as a ligand for CXCR4 and CXCR7 expressed by VSMCs and pericytes $)^{10,63,113}$. DVR ECs also express Slc14a1 and Aqp1 which encode the urea transporter B (UTB) $)^{10,11,112}$ and the water channel aquaporin $1\left(\mathrm{REFS}^{10,11,55}\right)$, both of which are required for urine concentration ${ }^{114,115}$ (FIG. 3c,d). These ECs also express Scin, which encodes scinderin - a protein that binds aquaporin 2 in a multiprotein complex in collecting duct epithelial cells, presumably to facilitate aquaporin 2 trafficking ${ }^{116}$. The co-expression of Aqp1 and Scin in DVR ECs suggests a similar interaction in the medullary endothelium ${ }^{10}$.

The osmolarity gradient establishes a hostile environment for cells of the renal medulla, especially for those in the renal papilla, where osmolarity is highest (corresponding to a condition of physiological hyperosmolarity in which the osmolarity is higher than in the systemic plasma $)^{117}$. Mouse DVR ECs can be separated into two main phenotypes according to their location in the renal papilla or in the outer or inner medulla ${ }^{10}$, and distinguished by the expression of hyperosmolarity-induced and of vasotone-regulatory genes ${ }^{10}$ (FIG. 3c). Renal papilla DVR ECs express hyperosmolarity-responsive genes, including target genes of the hyperosmolarity-inducible transcription factor NFAT5, such as S100a4 and S100a6 $\left(\right.$ REFS $\left.^{10,118}\right)$, whereas DVR ECs from the inner and outer medulla show enriched expression of Hpgd, which encodes a major enzyme involved in the catabolism 
S-nitrosylation

A post-translational

modification that results

from the biochemical reaction

between nitric oxide and a

cysteine thiol from a protein.

The covalent binding of the nitrosyl group to the protein

is reversible and regulates the activity of multiple proteins. of vasoactive prostaglandins, $E d n 1$, which encodes the vasoconstrictor endothelin 1, and Adipor2, which encodes a receptor for adiponectin that induces vasodilator effects ${ }^{119,120}$ (FIG. 3c). This expression pattern is consistent with the more prominent presence of smooth muscle-like pericytes in the outer medullary portion of the DVR and hence the greater responsiveness of this region to vasoactive factors, as compared with lower DVR portions ${ }^{9,121}$.

Unlike the DVR, AVR are fenestrated venous-like vessels (FIG. 3d). These vessels reabsorb water from the renal medullary interstitium that accumulates during urine concentration by the collecting ducts, the loop of Henle and the DVR, collecting it back into the general circulation in a manner akin to the function of lymphatic vessels ${ }^{30}$. In line with this role, AVR ECs express the venous transcription factor Nr2f2 (REFS ${ }^{10,11,64}$ ) and Plvap - probably to sustain their role in water reabsorption ${ }^{10,11,47,122}$ (FIG. 3c). AVR ECs also express Tek, encoding the angiopoietin Tie2 receptor, which is necessary for AVR formation during development. Deletion of Tek in mice triggers the rapid accumulation of fluid and cysts in the medullary interstitium and loss of medullary vascular bundles, and results in decreased urine concentrating ability ${ }^{30}$.

Similar to DVR, the AVR can be separated into two transcriptomically different EC populations located in the papilla and in the outer and inner medulla. Those in the papilla are characterized by the expression of hyperosmolarity-responsive genes (Cryab, Fxyd2 and Cd9 $\left(\right.$ REFS $\left.^{10,123,124}\right)$ ), glycolytic genes (Ldha, Aldoa and Gapd $h^{10,125,126}$ ), and Car2, which encodes the carbonic anhydrase 2 enzyme, the absence of which impairs urine concentration and triggers polyuria in mice ${ }^{127}$ (FIC. 3c). Papillary AVR ECs specifically express the $\mathrm{Na}^{+} / \mathrm{K}^{+}$ ATPase subunit-encoding gene Fxyd2, whereas an alternative subunit-encoding gene Fxyd6 is upregulated in AVR ECs in the outer and inner medulla ${ }^{10}$ (FIG. 3c).

The papillary portions of the AVR and DVR display distinct gene expression profiles, but share the expression of several hyperosmolarity-responsive genes, including Akr1b3, which encodes aldose reductase the rate-limiting enzyme of the polyol pathway that is responsible for the conversion of glucose into sorbitol, an inert organic osmolyte that is important for cell volume maintenance under conditions of hyperosmolarity ${ }^{117}$. They also express S100a6, as well as other genes - such as Fxyd5 (which encodes another $\mathrm{Na}^{+} / \mathrm{K}^{+}$ATPase subunit), Nrgn (which encodes the calmodulin-binding protein neurogranin) and Crip1 (which encodes cysteine-rich protein 1$)^{10,118}$ - that might potentially be linked to the hyperosmotic environment (FIC. 3c).

The renal medullary capillary plexus, which connects the DVR and AVR (FIG. 3a), is characterized by a Plvap-positive fenestrated endothelium and the enriched endothelial expression of genes that encode VEGF receptors, such as $K d r$, Flt1 and $N r p 1$, as well as genes involved in fatty acid transport and metabolism (Cd36 and Plpp3) $)^{10}$ (FIG. 3c). mRECs also include ECs from postcapillary venules, as well as angiogenic and interferon-activated EC populations, similar to the capillaries of the renal cortex ${ }^{10}$.

\section{REC heterogeneity and kidney disease}

Under physiological conditions the endothelium is quiescent - a state that is maintained in large part through the $S$-nitrosylation of proteins and transcription factors by eNOS-derived $\mathrm{NO}^{128,129}$. The activity of eNOS itself is regulated by shear stress ${ }^{130}$ and intracellular metabolites, such as the eNOS substrate, L-arginine and its cofactor, tetrahydrobiopterin ${ }^{131}$. Under particular conditions for example, in response to infection - this quiescent state can be switched off, inducing the activation of ECs and the recruitment of the immune cells. Redox signalling and in particular, uncoupling of the eNOS enzyme resulting in the production of superoxide instead of $\mathrm{NO}$ is critical to this activation process. The uncoupling of eNOS sets in motion a cascade that leads to remodelling of the endothelial surface layer and induces the expression of receptors that can interact with platelets and immune cells ${ }^{132}$. Although endothelial activation forms part of the host defence system, this molecular machinery can be inappropriately activated in disease conditions such as autoimmune disease or in the setting of cardiovascular risk factors or infection. Of note, heterogeneity exists in the response of RECs to injurious signals $^{133}$. For example, in atypical haemolytic uraemic syndrome, mutations in the complement inhibitory factor $\mathrm{H}$ are associated with reduced factor $\mathrm{H}$ binding to glomerular endothelial heparan sulfate ${ }^{134}$, thus inducing a glomerular thrombotic microangiopathy. Another example is chronic humoral allograft rejection in which the peritubular capillaries seem to be the primary target of injury ${ }^{135}$; the associated loss of the peritubular capillary network predicts the occurrence of kidney failure ${ }^{136}$. In the context of the COVID-19 pandemic, it is of note that $\mathrm{AKI}$ is frequently observed in patients with severe disease (affecting up to $50 \%$ of patients in intensive care units) ${ }^{137}$, in whom widespread EC dysfunction might promote disease escalation as a result of vascular leakage, coagulopathy and exacerbated inflammation ${ }^{138,139}$.

In addition to heterogeneity in the endothelial activation response, the response of RECs to environmental cues from the circulation may be site-specific. For example, gRECs from patients with type 1 diabetes mellitus demonstrate a dysregulated angiogenic response resulting in glomerular growth and secondary podocytopathy ${ }^{140,141}$. In ischaemic injury - in peritubular capillaries in particular - endothelial activation and sloughing off of ECs result in the so-called 'no-reflow' phenomenon, whereby perfusion is not restored even upon restoration of patency, resulting in tubular epithelial cell injury and $\mathrm{AKI}^{142}$. The clinical pathology induced by REC activation is discussed in detail elsewhere ${ }^{8}$.

The emergence of high-resolution techniques such as single-cell RNA-seq has provided new insights into the molecular regulation of endothelial phenotypic heterogeneity and the processes involved in kidney injury. A number of studies over the past few years, from our group and others, have advanced the concept that endothelial heterogeneity is interlinked with the intracellular metabolism ${ }^{3,6,10,143-145}$. As described below, the different microenvironments to which RECs are exposed help establish both their phenotypic diversity and metabolic specialization. 


\section{Metabolic specialization of RECs}

ECs exhibit an active metabolism even when quiescent to sustain processes such as energy production, biomass synthesis and redox homeostasis, which are necessary for the maintenance of vascular barrier integrity, vasoregulatory function, solute transport, and inhibition of thrombosis and vascular inflammation. For instance, quiescent ECs sustain high levels of FAO, which helps maintain vascular barrier integrity in part through the regeneration of $\mathrm{NADPH}$, which provides protection against reactive oxygen species (ROS) ${ }^{146}$. In line with this role, inhibition of FAO in ECs increases oxidative stress, endothelial barrier permeability, leukocyte infiltration ${ }^{146}$ and endothelial-to-mesenchymal transition ${ }^{147}$, suggesting that FAO is required for the maintenance of endothelial function and phenotype. RECs show different metabolic profiles and transcriptomes to ECs isolated from other organs in mice ${ }^{3,6}$. In particular, they are characterized by upregulation of genes involved in amino acid and pyrimidine biosynthesis as well as glucose metabolism ${ }^{6}$. Moreover, some metabolic genes are selectively enriched in arterial, capillary or venous ECs, indicative of intra-organ metabolic heterogeneity ${ }^{6}$. As discussed below, different microenvironmental conditions to which different REC populations are exposed might also affect their metabolic profiles, and support REC phenotypic heterogeneity as well as their response to disease stimuli.

\section{$R E C$ responses to changes in oxygen tension}

Although kidneys are the most perfused organs in the body, less than $10 \%$ of circulating oxygen is consumed during the passage of blood through the kidneys ${ }^{148}$. The kidney medulla is exposed to low oxygen tension, with a $\mathrm{pO}_{2}$ of $10-20 \mathrm{mmHg}$ (hypoxia) compared with $50 \mathrm{mmHg}$ in the kidney cortex ${ }^{117}$ (FIG. 4a). The oxygen

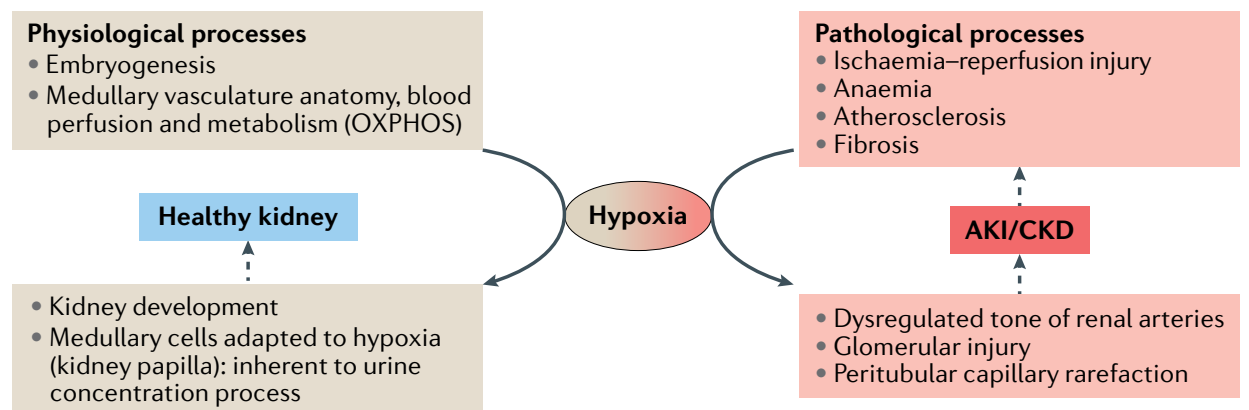

b Molecular and metabolic adaptation to hypoxia

Activation of HIF1 $\alpha$ and HIF2 $\alpha$

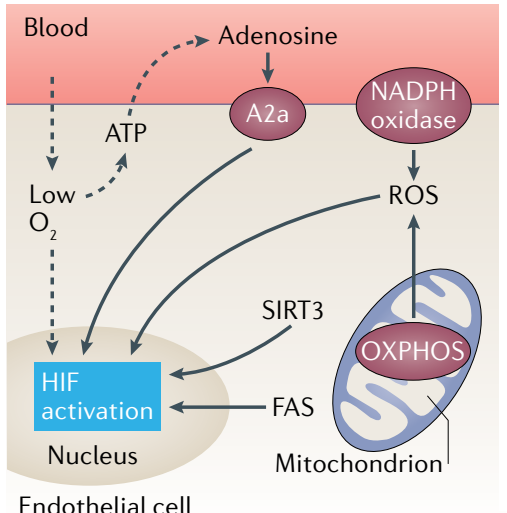

- HIF $1 \alpha$ is restricted to

papillary mRECs

- HIF2 $\alpha$ activation is renoprotective
Metabolic response to hypoxia

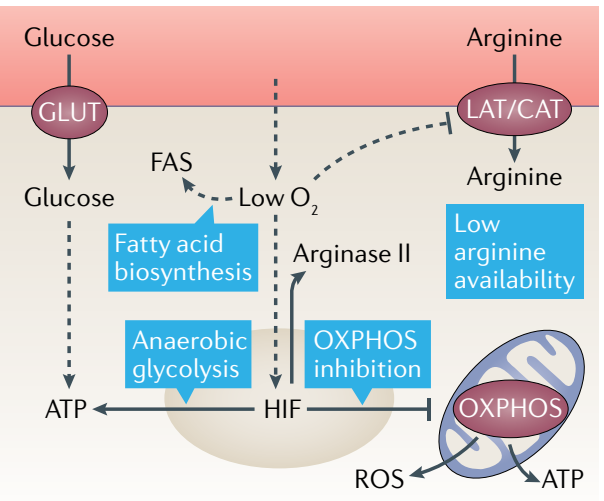

- Metabolic reprogramming

- eNOS uncoupling

- Endothelial dysfunction
SIRT3-FOXO3antioxidant pathway

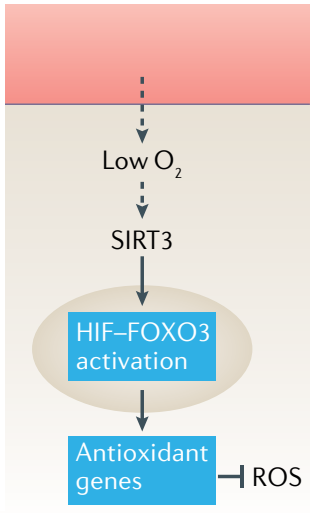

- Inhibition of glomerular endoMT - Inhibition of renal fibrosis

Fig. 4 | Exposure of the renal endothelium to changes in oxygen tension. a | The kidney is exposed to hypoxia under both physiological and pathological circumstances, such as in acute kidney injury (AKI) and chronic kidney disease (CKD). b Endothelial cells (ECs) adapt to hypoxia by stabilizing and activating the transcription factor hypoxia inducible factor (HIF) through the direct effects of low $\mathrm{O}_{2}$ levels, the release of ATP and downstream activation of the adenosine A2a receptors, the production of NADPH-derived and oxidative phosphorylation (OXPHOS)-derived reactive oxygen species (ROS), and as a consequence of sirtuin 3 (SIRT3) activation and the upregulation or activation of fatty acid synthase (FAS) (left panel). Exposure to hypoxia affects the metabolism of ECs by enhancing anaerobic metabolism and fatty acid biosynthesis while repressing aerobic metabolism (OXPHOS). Levels of the metalloenzyme arginase II are increased whereas arginine uptake is repressed, leading to low intracellular arginine availability, which results in endothelial nitric oxide synthase (eNOS) uncoupling and the production of ROS (central panel). ECs counteract ROS produced as a result of the exposure to hypoxia by activating a SIRT3-forkhead box 3 (FOXO3)-dependent antioxidant gene expression pathway (right panel). CAT, cationic amino acid transporter; endoMT, endothelial-to-mesenchymal transition; GLUT, glucose transporter; LAT, large neutral amino acid transporter. 
gradient that follows the corticopapillary axis is the consequence of several factors, including an arteriovenous oxygen shunt that results from the parallel arrangement of the AVR and DVR in the medulla, the limited blood flow to and within the medulla to minimize the washout of solutes, and the use of oxidative phosphorylation to produce the high levels of energy required for the $\mathrm{Na}^{+} / \mathrm{K}^{+}$ATPase to reabsorb $\mathrm{Na}^{+}$and to enable the proper functioning of other cell membrane solute transporters ${ }^{117}$. Thus, hypoxia is inherent to the urine concentration mechanism of the medulla ${ }^{10,117}$. It is also required for appropriate kidney development ${ }^{149}$. However, hypoxia can be detrimental and is considered a major cause of $\mathrm{AKI}^{150}$, and a risk factor for chronic kidney disease (CKD) ${ }^{151}$ (FIG. 4a). Kidney hypoxia can result from ischaemic events, such as can occur during kidney transplantation or as a result of abnormal renal perfusion due to peritubular capillary rarefaction, glomerular injury, atherosclerosis, dysregulation of arterial vascular tone, anaemia and impaired oxygen diffusion due to fibrosis ${ }^{152}$ (FIG. 4a). Within the vascular system, short-term exposure to hypoxia causes reversible modulation of vascular tone and blood flow, whereas long-term exposure results in irreversible remodelling of the vasculature and the surrounding tissues with VSMC proliferation and fibrosis ${ }^{153}$.

The cellular response to hypoxia depends on the inactivation of $\mathrm{Fe}^{2+}$-dependent oxygenases and 2 -oxoglutarate (2-OG)-dependent oxygenases ${ }^{152}$, and the subsequent activation of hypoxia-inducible transcription factor (HIF)-dependent and HIF-independent pathways. Exposure to hypoxia triggers the activation of both HIF1 $\alpha$ and HIF2 $\alpha$ in ECs ${ }^{154}$ (FIG. 4b). In the kidney, RECs widely express HIF2 $\alpha$ upon hypoxia, while protein expression of HIF1 $\alpha$ is limited to mRECs in the papilla ${ }^{155-157}$, where it probably stimulates glycolysis (FIG. 4b). Activation of HIF2 $\alpha$ in RECs in general mediates protection and recovery from ischaemic kidney injury by promoting erythropoiesis, and by suppressing renal inflammation, capillary rarefaction and fibrosis $^{156}$ (FIG. 4b). Exposure of RECs to hypoxia in the context of kidney disease might therefore induce different responses in gRECs and cRECs than in mRECs. For example, hypoxia promotes HIF1a-dependent proliferation and migration of cultured ECs ${ }^{158,159}$; however, in non-confluent conditions, cultured gRECs undergo mitochondria-dependent apoptosis upon exposure to hypoxia $^{155,160,161}$, suggesting a maladaptation of gRECs to hypoxia. Although gRECs seem to be quite resistant to hypoxia in vivo, probably owing to the paracrine effect of podocyte-derived VEGF ${ }^{161}$, hypoxia can induce a progressive loss of the tight junction proteins occludin and ZO-1 in gRECs in a HIF $2 \alpha$-dependent manner, ultimately increasing endothelial barrier permeability ${ }^{162}$. Little is known about the response of mRECs to hypoxia. mRECs in the AVR and DVR in particular are exposed to low oxygen tension in the papilla under physiological conditions, and the Epas 1 regulon (encoding HIF2 $\alpha$ ) is upregulated in mRECs upon water deprivation, probably in response to an increase in hypoxia driven by the urine concentration process $^{10}$.
Metabolic adaptation of ECs to changes in oxygen tension. Under normoxic conditions, ECs rely primarily on glycolysis for ATP production rather than mitochondrial oxidative phosphorylation ${ }^{163}$. In response to hypoxia, these metabolic responses are exacerbated, with further enhancement of glycolysis and suppression of mitochondrial respiration (FIG. 4b), explaining why ECs are resistant to hypoxia as long as glucose remains available ${ }^{164}$. Upon exposure to acute hypoxia such as an ischaemic event, ECs show a rapid increase in mitochondrial and/or $\mathrm{NAD}(\mathrm{P}) \mathrm{H}$ oxidase-derived ROS, which stabilizes HIF1 $\alpha$ and enables higher glycolytic flux ${ }^{164}$ - responses that are consistent with a HIF1 $\alpha$-induced upregulation of glucose metabolism and a downregulation of mitochondrial activity ${ }^{164,165}$ (FIG. 4b). Moreover, metabolic pathway analyses of ECs exposed to chronic hypoxia, such as can occur in the medulla or in the context of CKD, revealed a HIF2 $\alpha$-dependent upregulation of glycolytic genes ${ }^{166}$. Interestingly, some glycolytic genes such as Eno1 and Aldoa, which encode the enzymes enolase 1 and aldolase A that are necessary to produce ATP and pyruvate from glucose, were upregulated to a greater extent in mRECs than in cRECs and gRECs ${ }^{10}$. More specifically, mRECs from the papillary portion of the AVR that is the portion of the renal vascular bed that is most exposed to hypoxia - showed the highest expression of the glycolytic genes Aldoa, Ldha and Gapdh among all mRECs in mice ${ }^{10}$. Thus, papillary mRECs might demonstrate higher anaerobic glycolytic flux than other RECs as a result of their hypoxic microenvironment. Similarly, medullary epithelial cells have a higher capacity for anaerobic glycolytic ATP production than proximal tubular cells ${ }^{117}$. mRECs also upregulate several glycolytic genes upon water deprivation, concurrent with the increased HIF $2 \alpha$ activity mentioned above ${ }^{10}$.

In ECs, HIF2 $\alpha$ is upregulated in part following activation of the mitochondrial $\mathrm{NAD}^{+}$-dependent deacetylase sirtuin 3 (SIRT3) (REF. ${ }^{167}$ ) (FIG. 4b). Loss of SIRT3 impairs hypoxic signalling in ECs, and results in defective angiogenesis and microvascular dysfunction, secondary to a metabolic switch from oxygen-independent glycolysis to mitochondrial respiration. This metabolic switch is associated with a decrease in the expression of 6-phosphofructo-2-kinase (PFKFB3), an enzyme that acts as a positive regulator of glycolysis and ROS formation $^{167}$ (FIG. 4b). Upon hypoxia, SIRT3 upregulates mitochondrial antioxidant enzymes in a manner dependent on FOXO3 $\left(\right.$ REF. $\left.^{168}\right)$ - a transcription factor that is also upregulated by HIFla ${ }^{169}$ (FIG. 4b). Interestingly, the SIRT3-FOXO3 antioxidant pathway is operational in gRECs, preventing endothelial-to-mesenchymal transition and kidney fibrosis in an animal model of angiotensin-II-induced hypertension ${ }^{170}$ (FIG. 4b). Pharmacological approaches that increase SIRT3 also limit cisplatin-induced AKI by protecting against tubular injury and by improving kidney function ${ }^{171}$. By contrast, Sirt3-knockout mice exhibit more severe AKI, although the contribution of RECs to these effects has not been determined ${ }^{171}$. Whether this SIRT3-FOXO3 antioxidant pathway is also involved in the physiological response of mRECs to hypoxia in the medulla remains to be determined. 
The metabolism of fatty acids is also affected by oxygen availability, since hypoxia triggers an increase in the expression and activity of fatty acid synthase (FAS), a key rate-controlling enzyme of the fatty acid biosynthesis pathway, resulting in a reduction of the malonyl-coA pool and an augmentation of palmitate levels in ECs ${ }^{172}$ (FIG. 4b). In human pulmonary artery ECs, inhibition of FAS leads to impairment of HIF1a stabilization, and subsequent HIF1 $\alpha$-mediated changes in glucose transport and metabolism and to the restoration of eNOS function, suggesting that the inhibition of fatty acid synthesis may be beneficial for EC function in hypoxia ${ }^{172}$ (FIG. 4b). In the kidney, Fasn - which encodes FAS - was upregulated in an experimental model of chronic kidney failure and contributed to hypertriglyceridaemia ${ }^{173}$. Upregulation of Fasn and other hypoxia-responsive genes was also observed in the kidney cortex of a mouse model of sickle cell anaemia that exhibited progressive glomerular and tubular damage ${ }^{174}$. Altered lipid metabolism is a characteristic of proteinuric CKD and both clinical and experimental evidence support the notion that altered lipid metabolism might contribute to the pathogenesis and progression of kidney disease ${ }^{175}$. Nonetheless, the role of RECs in dysregulated fatty acid metabolism in the context of kidney disease remains to be further clarified.

Hypoxia also induces the upregulation of arginase II in a manner dependent on the activation of HIF $2 \alpha^{176}$ or HIF $1 \alpha^{177}$, and decreases the synthesis and transport of
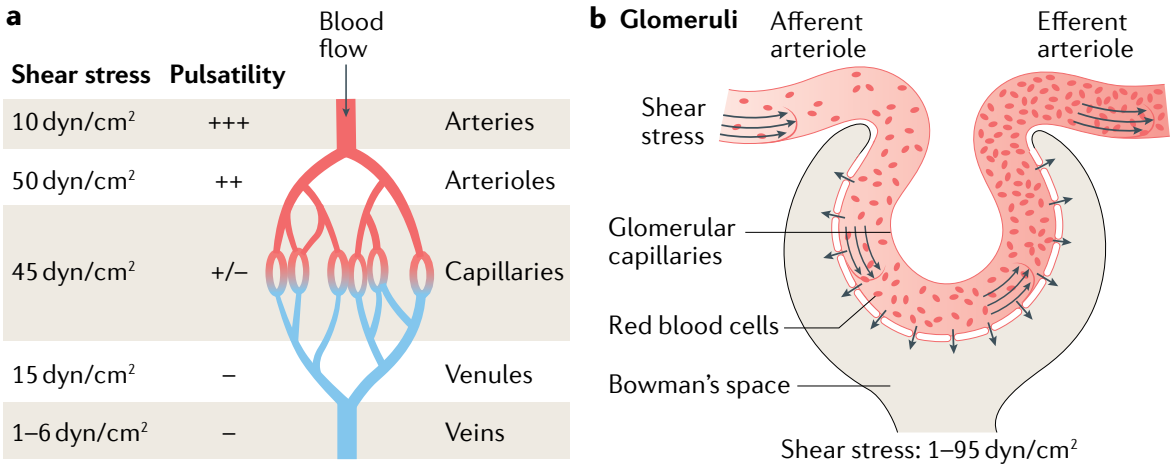

c Renal arteries and arterioles

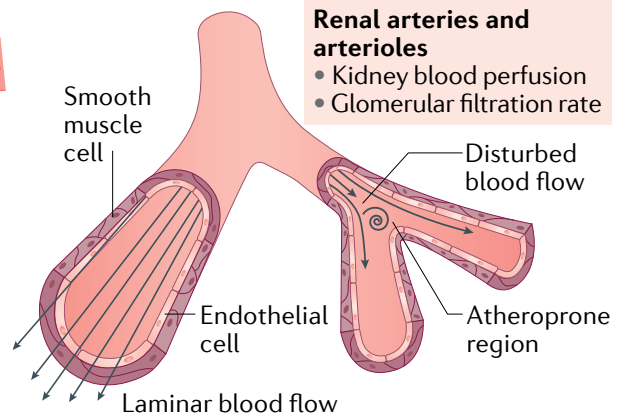

\section{d Molecular and metabolic adaptation to laminar blood flow}

Glycolysis inhibition and glycocalyx biosynthesis

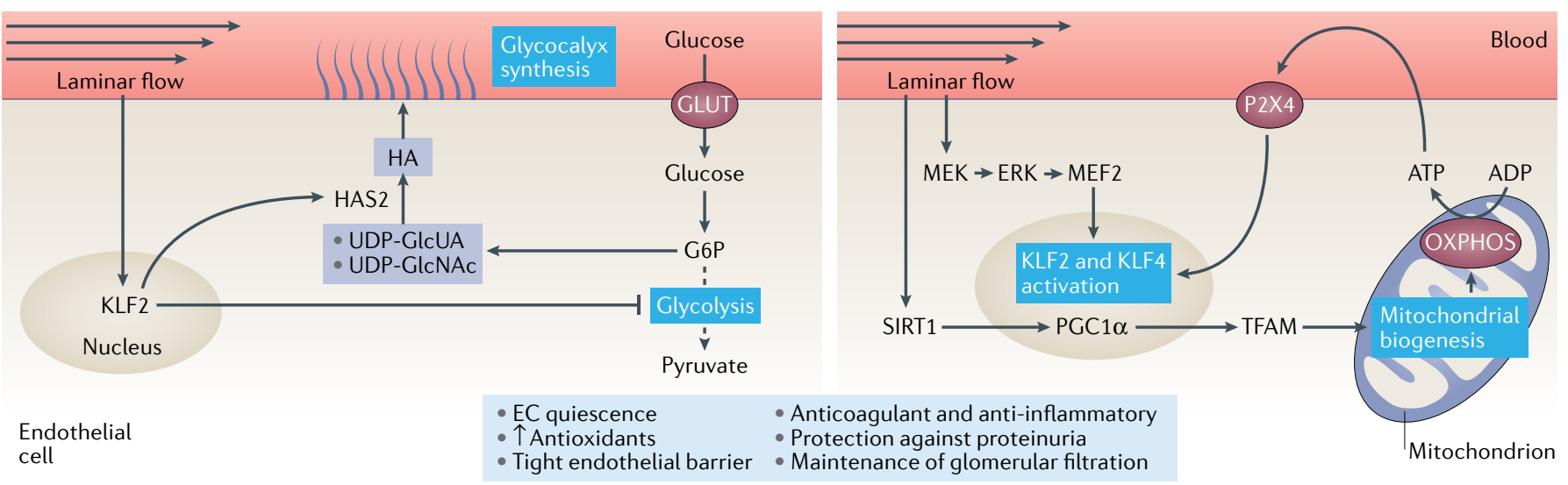

e Molecular and metabolic adaptation to disturbed blood flow

Repression of laminar flow-induced vasculo-protective factors

ROS/NF-KB-dependent glycolysis stimulation and glycocalyx degradation
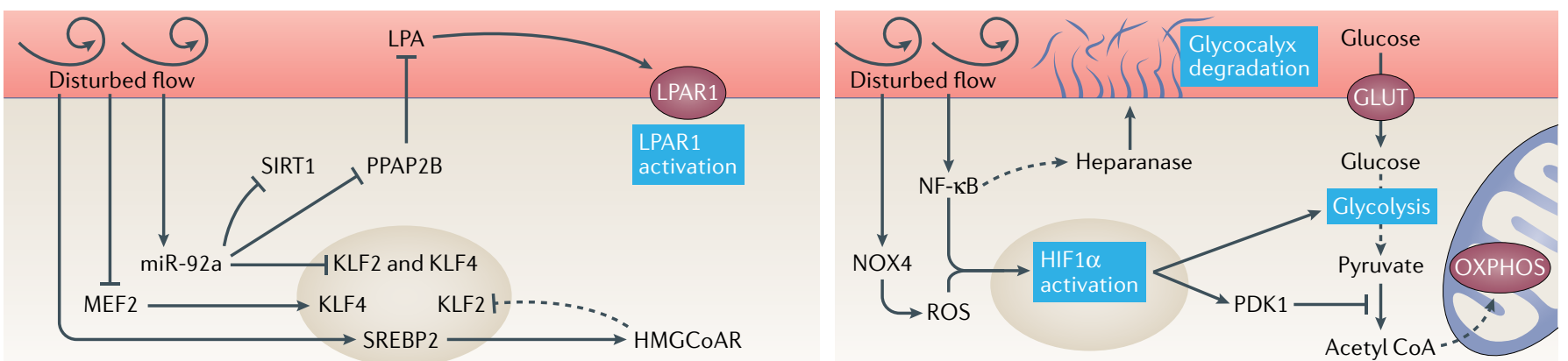

Endothelial cell $\begin{array}{ll}\text { - EC activation } & \text { Pro-coagulant and } \\ \text { - Oxidative stress } & \text { pro-inflammatory effects } \\ \text { - Impaired endothelial barrier } & \text { - Atherosclerotic renal artery stenosis }\end{array}$
Glycolysis and mitochondrial metabolism uncoupling 
its substrate, arginine, in ECs ${ }^{178,179}$ (FIG. 4b). Arginase II is a metalloenzyme that is particularly expressed in the kidneys and catalyses the hydrolysis of L-arginine into urea and $\mathrm{L}$-ornithine. The increase in arginase II activity lowers arginine bioavailability, which dampens eNOS activity, decreasing endothelial NO production and triggering eNOS uncoupling, ultimately leading to the production of ROS and nitrosative stress ${ }^{176}$. These steps are critical in promoting endothelial dysfunction, diabetic kidney disease and kidney inflammation in the context of diet-induced obesity ${ }^{180,181}$. Under physiological conditions, arginase II is mainly expressed in the outer medulla, suggesting that this metabolic adaptation probably does not occur in the mRECs that are most exposed to hypoxia ${ }^{181}$.

Finally, exposure of papillary mRECs to acute hypoxia triggers the release of purines and ATP, together with UTP and UDP, in the extracellular space ${ }^{182-184}$. ATP activates endothelial $\mathrm{P} 2 \mathrm{Y}$ receptors, resulting in $\mathrm{NO}$ production, vasodilation and increased tissue perfusion ${ }^{185}$.

4 Fig. 5 | Response of the renal endothelium to changes in blood flow and shear stress. a Arteries, arterioles, capillaries, venules and veins are exposed to different types of blood flow as defined by its pulsatility and the level of shear stress. b | Glomerular capillaries are exposed to high shear stress as a result of high blood flow and pressure together with increasing blood viscosity due to the filtration process. $\mathrm{c}$ |Some regions of renal arteries and arterioles - which control kidney blood perfusion and glomerular filtration rate - are exposed to laminar blood flow, whereas other regions - particularly regions of vessel bifurcation - are exposed to disturbed blood flow. Regions of disturbed blood flow are more likely to develop atherosclerotic lesions. $\mathbf{d}$ | Endothelial cells (ECs) exposed to laminar blood flow inhibit glycolytic metabolism in a KLF2-dependent manner and divert glycolytic intermediates to pathways involved in glycocalyx biosynthesis (left panel). Laminar flow also induces SIRT1-PGC1 $\alpha$-TFAM-mediated mitochondrial biogenesis, and stimulates the production of oxidative phosphorylation (OXPHOS)derived ATP, which activates purinergic $\mathrm{P} 2 \mathrm{X} 4$ receptors and further induces the activation of KLF2 and KLF4 transcription factors. Moreover, Krüppel-like factor 2 (KLF2) and KLF4 are upregulated in response to MEK5-ERK5 pathway activation (right panel). Laminar blood flow promotes EC quiescence, the production of antioxidants, the formation of a tight endothelial barrier and the acquisition of an anticoagulant and anti-inflammatory phenotype, and protects against proteinuria while maintaining glomerular filtration. e | Disturbed blood flow represses the protective pathways induced by laminar blood flow. Induction of microRNA (miR)-92a by disturbed flow represses the endothelial expression of KLF2, KLF4 and SIRT1 and PPAP2B, releasing the inhibitory effect of PPAP2B on circulating lysophosphatidic acid (LPA). Binding of LPA to its receptor LPAR1 induces pro-inflammatory signalling. The induction of SREBP2 upregulates HMGCoA reductase, which increases intracellular cholesterol levels, and further upregulates miR-92a. Hypermethylation of the KLF4 promoter prevents MEF2 binding and subsequent KLF4 transcription (left panel). ECs exposed to disturbed blood flow also demonstrate an uncoupling of glycolysis from mitochondrial metabolism, with an upregulation of glycolysis. Disturbed flow also induces the production of NOX4-derived reactive oxygen species (ROS) and activates nuclear factor $\kappa B(N F-\kappa B)$, leading to the upregulation and stabilization of hypoxia inducible factor $1 \alpha$ (HIF1 $\alpha$ ). Activation of the NF- $\kappa B$ pathway has been linked to heparanase activity and consequent glycocalyx degradation (right panel). Disturbed blood flow ultimately triggers EC activation and oxidative stress, impairs the endothelial barrier, induces a procoagulant and proinflammatory EC phenotype, and favours the development of atherosclerotic renal artery stenosis. ERK, extracellular signal regulated kinase; G6P, glucose-6-phosphate; GLUT, glucose transporter; HA, hyaluronan; HAS2, hyaluronan synthase 2; HMGCoAR, hydroxymethylglutaryl CoA reductase; MEF2, myocyte enhancer factor-2; MEK, mitogen-activated protein kinase kinase; NOX4, NADPH oxidase 4; PDK1, pyruvate dehydrogenase kinase 1; PGC1 $\alpha$, peroxisome proliferator-activated receptor gamma coactivator-1a; PPAP2B, phosphatidic acid phosphatase type 2B; SIRT1, sirtuin 1; SREBP2, sterol regulatory element binding protein 2; TFAM, mitochondrial transcription factor A; UDP-GlcNAc, uridine diphosphate- $N$-acetylglucosamine; UDP-GlcUA, uridine diphosphate-glucuronic acid.
ATP also forms adenosine following metabolism of the ATP by ectoenzymes ${ }^{185,186}$. Importantly, hypoxia triggers a HIF $2 \alpha$-dependent upregulation of adenosine A2a receptor (encoded by ADORA2A) in $\mathrm{ECs}^{187,188}$, the activation of which increases HIF1 $\alpha$ protein synthesis, further promoting glycolytic gene expression and glycolytic flux ${ }^{187}$ (FIG. 4b). In most instances, activation of A2a and $\mathrm{A} 2 \mathrm{~b}$ receptors expressed by ECs and VSMCs mediates the vasodilator effects of adenosine released during hypoxia ${ }^{185}$. In the kidney, different adenosine receptors are present in the different parts of the vasculature ${ }^{189}$, and extracellular ATP and adenosine exert key roles in the regulation of renal haemodynamics and the microcirculation ${ }^{185,190}$. In the medulla, adenosine is produced in the medullary thick ascending limb of the loop of Henle (TALH) after oxidative stress ${ }^{191}$ and acts as a vasodilator, inducing an increase in medullary blood flow via a mechanism that may involve DVR mRECs ${ }^{192}$. However, contrary to its effects in most other vessels, adenosine-mediated activation of A2a receptors, which are particularly expressed in the afferent arterioles, triggers vasoconstriction of the renal vasculature, thereby potentially affecting renal blood flow and glomerular filtration ${ }^{185,193}$. Of note, a role for purinergic receptors in CKD progression has been identified ${ }^{194}$.

\section{REC responses to changes in shear stress}

ECs are constantly exposed to a force of stretch induced by the pulsatility of blood flow and a frictional force parallel to the vessel wall, the fluid shear stress ${ }^{195}$. These cells are equipped to sense these forces and to transduce them into biochemical signals that can affect vessel homeostasis by regulating vascular tone and EC remodelling, which adjusts the blood flow to meet tissue requirements ${ }^{195}$. Interestingly, ECs from different portions of the vascular bed are exposed to specific flow types and respond accordingly ${ }^{195}$ (FIG. 5a). In arteries and arterioles, blood flow is highly pulsatory, whereas in capillaries it is of similar magnitude but less pulsatory, and in venules and veins blood flow is about threefold to tenfold lower and pulsatility is minimal ${ }^{195}$. In the kidney, the vasculature of the cortex receives $>94 \%$ of renal blood flow ${ }^{196}$ suggesting that the medullary vasculature is exposed to a relatively low shear stress environment. By contrast, gRECs are exposed to a relatively high shear stress environment (estimated to be from $1 \mathrm{dyn} / \mathrm{cm}^{2}$ to as high as $95 \mathrm{dyn} / \mathrm{cm}^{2}$ ), as a result of high blood flow and pressure combined with increasing blood viscosity as a result of the filtration process ${ }^{197}$ (FIG. 5b). Exposure of gREC to shear stress is critical since it prevents platelet aggregation, in part by inducing the conformational unfolding of the blood glycoprotein von Willebrand factor (vWF) which enhances its susceptibility to cleavage by the ADAMTS13 metalloprotease ${ }^{198,199}$. The importance of this phenomenon to gREC health is demonstrated by the pro-thrombotic phenotype observed in Shiga toxin-associated haemolytic uraemic syndrome. Shiga toxin promotes the secretion of vWF by gRECs and the formation of ultra-large vWF multimers that are resistant to cleavage by ADAMTS13 and induce thrombotic microangiopathy in glomeruli and the kidney microvasculature ${ }^{200}$, eventually leading 
to AKI. In thrombotic thrombocytopenic purpura, low ADAMTS13 activity results in a similar outcome ${ }^{199}$.

The importance of shear stress is also illustrated by the development of atherosclerotic lesions in renal arteries, which can result in renal artery stenosis - the single largest cause of secondary hypertension ${ }^{201,202}$. These lesions develop in atheroprone regions in the arteries and arterioles that are exposed to lower laminar shear stress than other regions, such as regions of arterial bifurcation, where blood flow is typically disturbed $^{201}$ (FIG. 5c).

Effects of laminar shear stress on ECs. Laminar shear stress induces upregulation of the transcription factors Krüppel-like factor 2 (KLF2) and KLF4 in ECs, in part through the release of ATP and subsequent activation of $\mathrm{P} 2 \mathrm{X} 4$ purinergic receptors ${ }^{203}$, and in part via activation of the MEK5-ERK5-MEF2 signalling pathway ${ }^{204,205}$ (FIG. 5d). In the kidney, Klf2 and Klf4 together with the KLF4 target gene, Thbd (which encodes thrombomodulin), have been reported to be markers of gRECs derived from the efferent arterioles in adult mice ${ }^{10,11}$ (FIG. 3b). The location of these flow-responsive markers is consistent with the fact that RECs located at the immediate exit site of glomeruli are exposed to a high laminar shear stress, potentially related to the high blood viscosity of this region. The upregulation and activation of KLF2 mediates EC quiescence, characterized by an increase in the expression of VE-cadherin and $\beta$-catenin to aid the maintenance of a tight vascular barrier ${ }^{206}$, the alignment of ECs in the direction of flow ${ }^{207}$, the inhibition of inflammation and maintenance of an anti-atherogenic phenotype, an upregulation of antioxidants and a decrease in vascular tone secondary to the endothelial production of NO and prostacyclins ${ }^{208-210}$ (FIG. $5 \mathrm{~d}$ ). Activation of KLF2 in gRECs protected them against injury and disease progression in animal models of $\mathrm{CKD}^{211,212}$. Accordingly, KLF2 is upregulated in the glomerular endothelium in response to shear stress in vitro, where it promotes an anticoagulant and antiinflammatory phenotype and triggers an endothelialdependent decrease in podocyte barrier resistance, required for proper filtration function ${ }^{204}$. The upregulation of gREC KLF2 secondary to glomerular hyperfiltration conferred protection against EC dysfunction and attenuated the progression of CKD in a model of unilateral nephrectomy ${ }^{212}$. Conversely, loss of endothelial KLF2 exacerbated glomerular hypertrophy and proteinuria in a model of streptozotocin-induced diabetic kidney disease ${ }^{211}$. Endothelial KLF4 is also renoprotective in $\mathrm{AKI}^{213}$. EC-specific loss of KLF4 exacerbated kidney injury in a model of ischaemic AKI by promoting EC acquisition of a pro-inflammatory phenotype ${ }^{213}$. However, it is worth noting that KLF2 and KLF4 have context-specific roles in the endothelium ${ }^{214}$. For instance, they can promote the activation of ECs and the formation of lesions leading to cerebral cavernous malformations in development ${ }^{214,215}$.

Exposure to laminar shear stress reduces glucose uptake $^{216}$ and stimulates mitochondrial biogenesis in $\mathrm{ECs}^{217-219}$. KLF2 activation downregulates the expression of PFKFB3 together with that of other glycolytic genes such as $H K 2$ (which encodes hexokinase 2) and PFK1 (which encodes phosphofructokinase 1 (PFK1)), resulting in a decrease in glycolysis ${ }^{216}$, and the shuttling of available early glycolytic intermediates to the hexosamine and glucuronic acid biosynthetic pathway for UDP-GlcUA and UDP-GlcNAc synthesis, respectively, which are the limiting substrates of hyaluronan synthase (HAS2) $)^{133,220-222}$. KLF2 also induces the expression and membrane translocation of HAS2, and the consequent synthesis of the glycocalyx component hyaluronan ${ }^{133,222}$. Thus, ECs exposed to laminar flow display a much thicker glycocalyx than ECs exposed to disturbed flow $^{133}$ (FIG. 5 d,e). EC-specific deletion of Has 2 has profound effects on the kidney, including impairment of the glycocalyx structure of capillary gRECs, disruption of the glomerular endothelial fenestrations ${ }^{40}$, albuminuria indicative of filtration barrier dysfunction, disturbed glycocalyx-dependent angiopoietin 1 signalling, and abnormal podocyte structures as a result of abnormal EC-podocyte crosstalk, resulting in glomerular capillary rarefaction and glomerulosclerosis ${ }^{40}$.

Mitochondrial respiration and ATP generation are also increased in ECs under unidirectional flow compared with those exposed to disturbed flow ${ }^{223,224}$. Blockade of mitochondrial ATP generation inhibits shear stress-induced ATP release whereas by contrast, inhibition of glycolysis has no effect, suggesting that EC mitochondrial respiration is required for purinergic receptor activation, which in turn induces KLF2 expression in response to shear stress ${ }^{203,218,224}$. Furthermore, mitochondrial biogenesis is upregulated in response to shear stress, owing to the activation of a SIRT1-PGC1aTFAM signalling cascade ${ }^{217,219}$, whereas the expression of antioxidant genes, such as haem oxygenase 1 and glutaredoxin 1, are increased to protect ECs from ROS 225,226 (FIG. 5d). Inhibiting the electron transport chain in ECs exposed to laminar flow resulted in EC inflammation, suggesting that mitochondrial respiration prevents EC activation $^{223}$. In the kidney, activation of the 5-HT1F receptor to stimulate mitochondrial biogenesis in RECs may reduce vascular rarefaction and promote recovery from injury as has been shown in a model of $\mathrm{AKI}^{227}$.

KLF4 also induces the upregulation of cholesterol-25hydroxylase $(\mathrm{CH} 25 \mathrm{H})$ and liver $\mathrm{X}$ receptor upon exposure to an atheroprotective pulsatile shear stress ${ }^{228}$. $\mathrm{CH} 25 \mathrm{H}$ catalyses the production of 25-hydroxycholesterol, which prevents the activation of sterol regulatory element binding protein 2 (SREBP2), an important mediator in the EC response to disturbed blood flow (see below) ${ }^{229,230}$. Hence, modulation of KLF2 and KLF4 expression and activation by laminar shear stress and their consequent metabolic responses may have a critical role in the maintenance of REC quiescence and glomerular filtration (FIG. 5d).

Effects of a disturbed blood flow on ECs. ECs exposed to disturbed blood flow such as in arterial bifurcations or curvatures are activated and exhibit a pro-inflammatory and atherogenic phenotype ${ }^{231}$ (FIG. 5e). Thus, the activation of arterial and afferent arteriolar RECs located in such atheroprone regions might promote the development of atherosclerotic renal artery stenosis. 
KLF4 expression is repressed by disturbed blood flow, with increased methylation of the KLF4 promoter region preventing MEF2 binding and subsequent KLF4 transcription $^{232}$. Furthermore, microRNA (miR)-92a, which is induced in atheroprone regions in response to low shear stress, represses endothelial expression of KLF2, KLF4 and SIRT1, and downregulates phosphatidic acid phosphatase type $2 \mathrm{~B}$ (PPAP2B) ${ }^{216,233,234}$. Under conditions of normal laminar shear stress, PPAP2B dephosphorylates circulating lysophosphatidic acid (LPA), preventing its binding to the LPAR1 receptor, which otherwise induces pro-inflammatory signalling ${ }^{233}$ (FIG. 5e). Endothelial loss of PPAP2B leads to exacerbated local and systemic inflammation associated with an increase in endothelial permeability ${ }^{235}$. LPA signalling is involved in kidney disease through the induction of ROS, inflammatory cytokines and fibrosis ${ }^{236}$.

Endothelial exposure to low shear stress and disturbed flow induces the expression of glycolytic enzymes and of pyruvate dehydrogenase kinase 1 (PDK1), thereby uncoupling glycolysis from mitochondrial metabolism and decreasing mitochondrial respiration in $\mathrm{ECs}^{223,237}$ (FIG. 5e). Mechanistically, disturbed flow induces the production of $\mathrm{NAD}(\mathrm{P}) \mathrm{H}$ oxidase 4 (NOX4)-derived ROS and activates NF- $\kappa \mathrm{B}$, leading to upregulation and stabilization of HIF1 $\alpha$ by preventing its degradation ${ }^{223,237}$. Activation of the NF- $\kappa \mathrm{B}$ pathway has been linked to heparinase activity and consequent glycocalyx degradation ${ }^{238}$. In line with these findings, activation of $\mathrm{NF}-\kappa \mathrm{B}$ is repressed by chronic shear stress in the glomerular endothelium ${ }^{239}$. HIF1 $\alpha$ activation in response to disturbed flow enhances arterial EC proliferation and expression of inflammatory markers, whereas inhibition of glycolysis prevents these responses ${ }^{223,237}$. Moreover, activation of mitochondrial oxidative phosphorylation prevents the pro-inflammatory phenotype induced by disturbed flow in arterial $\mathrm{ECs}^{223}$.

Disturbances in low shear stress also induce activation of the pro-inflammatory transcription factors YAP and TAZ, whereas laminar shear stress inhibits these in an integrin-dependent manner ${ }^{240,241}$. Activation of YAP and TAZ modulates EC metabolism by stimulating glycolysis and mitochondrial activity in a MYC-dependent manner ${ }^{242}$, and by upregulating glutaminolysis ${ }^{243}$. Conversely, the glycolytic enzyme PFK1 stimulates YAP and TAZ activity in a positive feedback loop ${ }^{244}$. YAP and TAZ are mechanoregulators of the TGF $\beta-S M A D$ signalling pathway in the kidney. They have been demonstrated to promote kidney fibrosis in an experimental model of unilateral ureteral obstruction, although the role of RECs in this fibrotic response as a result of endothelial-to-mesenchymal transition has been poorly investigated $^{245,246}$.

Interestingly, ECs exposed to a disturbed blood flow also show activation of SREBP2, which upregulates genes involved in cholesterol synthesis including the rate-limiting enzyme of the mevalonate pathway HMG-CoA reductase (FIG. 5e), and decreases cholesterol efflux $^{230,247}$, increasing the intracellular level of cholesterol in $\mathrm{ECs}^{230,247}$. Interestingly, inhibition of HMG-CoA reductase by statins induces an endothelial increase in KLF2 expression, and reduces pro-inflammatory signalling by NF- $\kappa \mathrm{B}, \mathrm{HIF} 1 \alpha$ and YAP-TAZ, thereby inducing an EC-like response to laminar flow ${ }^{241,248,249}$. Furthermore, SREBP2 activation promotes the transcription of miR-92a, upregulates the expression of NOX2, which induces production of ROS, and increases the expression of the NLRP3 inflammasome, ultimately promoting endothelial inflammation and atherosclerosis $^{216,230}$. Thus, SREBP2 may be one of the key drivers in the renal arterial and afferent arteriolar endothelial response to a disturbed flow.

\section{REC responses to changes in osmolarity}

The kidneys can produce urine of widely varying osmolarity depending on hydration status. The countercurrent multiplier of the loop of Henle generates a medullary osmolarity gradient that underlies the urine concentration mechanism and determines the final urine osmolarity. Briefly, the TALH - a tubule segment that is mostly impermeable to water - actively shuttles $\mathrm{NaCl}$ from the filtrate to the medullary interstitium, establishing a $20 \mathrm{mOsm} / \mathrm{kg} \mathrm{H}_{2} \mathrm{O}$ osmolality difference across the ascending and descending flow ${ }^{250}$ (FIG. 6a). As an osmotic response, water is reabsorbed through the thin descending limb of Henle (TDLH), thereby increasing filtrate osmolarity ${ }^{250}$. As this filtrate progresses from the TDLH to the TALH, active reabsorption of $\mathrm{NaCl}$ by the TALH re-establishes the $20 \mathrm{mOsm} / \mathrm{kg}$ $\mathrm{H}_{2} \mathrm{O}$ osmolality difference between the TALH and interstitium, further increasing medullary interstitium osmolarity ${ }^{250}$. The multiplication of these small osmolality differences between countercurrent flows leads to a large corticomedullary osmolality gradient (a countercurrent multiplier $)^{250}$. At the level of the vasa recta, water efflux facilitated by aquaporins occurs in parallel with the reabsorption of urea and $\mathrm{NaCl}$ within the DVR, driven by the difference between the blood and medullary osmolarity, resulting in an increased blood osmolarity towards the papilla ${ }^{250}$ (FIG. 6a). By contrast, the highly fenestrated AVR reabsorb medullary water and release $\mathrm{NaCl}$ into the interstitium, since the blood coming from the papilla is of higher osmolarity than the medullary interstitium $^{250}$ (FIG. 6a). This countercurrent exchange between the DVR and AVR maintains the medullary osmolarity gradient created by the countercurrent multiplier system (FIG. 6a). The high osmolarity of the medulla is also sustained by the collecting ducts, which actively export urea within the inner medullary interstitium, while concentrating urine according to the medullary osmolarity gradient via aquaporin-facilitated water transport. As a consequence, medullary cells including mRECs are exposed to extreme levels of hyperosmolarity, especially under conditions of dehydration wherein osmolality can rise as high as $1,400 \mathrm{mOsm} / \mathrm{kg}$ in humans ${ }^{250}$ (FIG. 6a). As described below, available evidence suggests that mRECs have adapted to these extreme conditions by activating protective mechanisms and developing a specific metabolic profile ${ }^{10}$. Of note, other (R)ECs can be exposed to hyperosmolar conditions as a consequence of hyperglycaemia in the context of diabetes mellitus ${ }^{251}$.

The response of ECs to conditions of hyperosmolarity has been poorly investigated, with most of the 


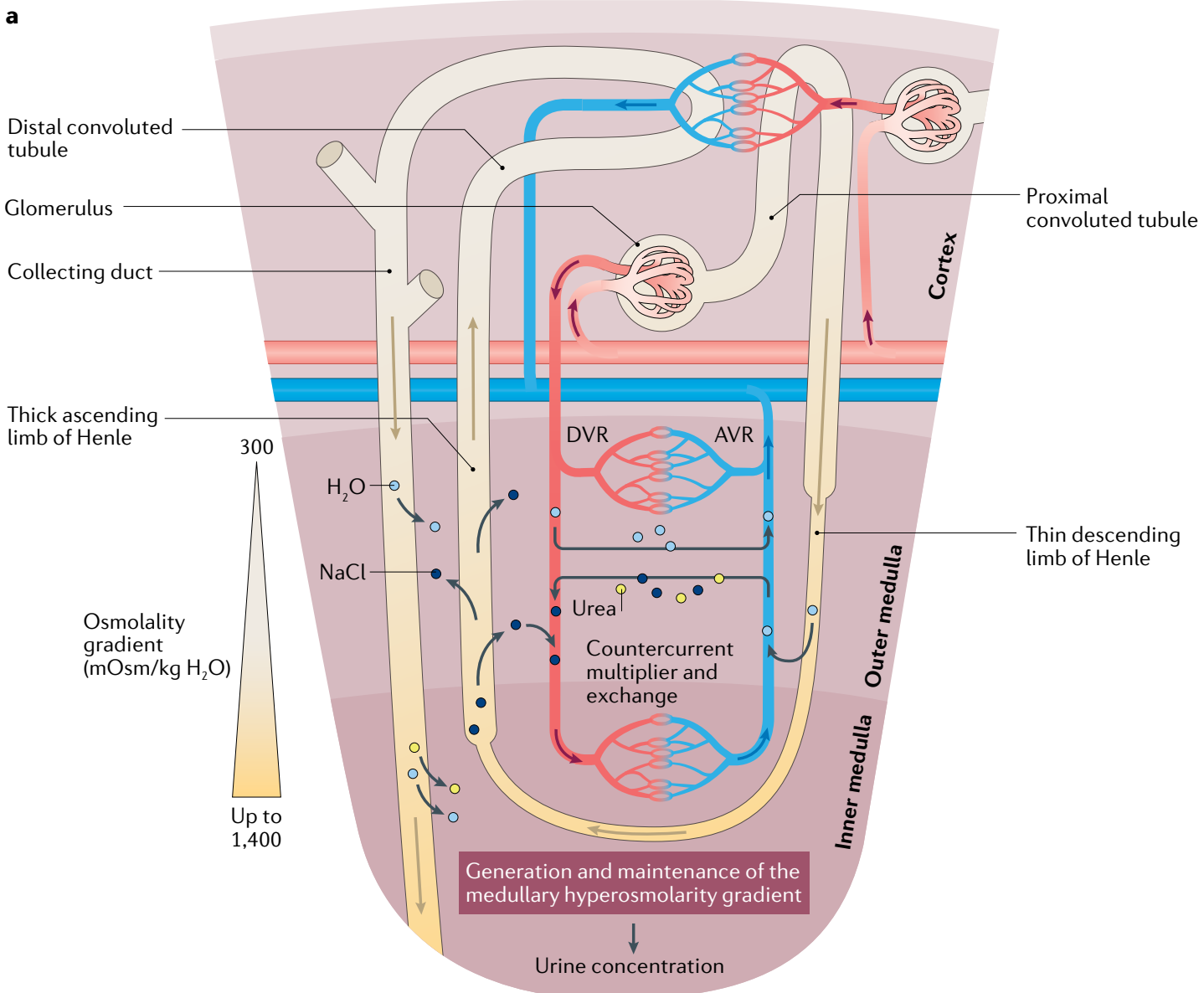

b Molecular and metabolic adaptation to hyperosmolarity

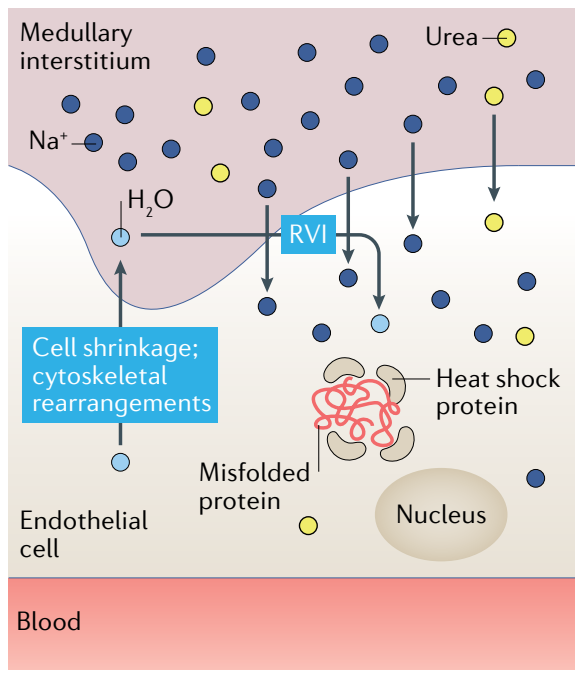

Early response

e.g. switch from diuresis to anti-diuresis
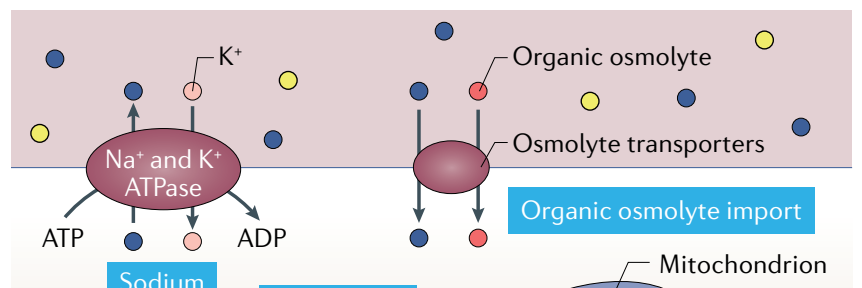

export

Glycolysis and organic osmolyte synthesis

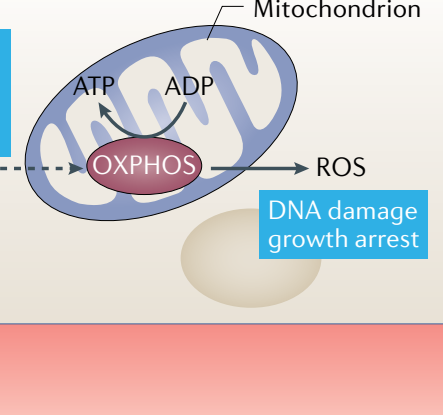

Chronic adaptation

e.g. situations of prolonged dehydration or papillary ECs

research in this field focusing on renal epithelial cells, influx of $\mathrm{Na}^{+}$ions for maintenance of cell volume. These in which hyperosmolarity triggers cell cycle arrest, the production of ROS and DNA damage ${ }^{252}$. Specifically, the epithelial response to hyperosmolarity is characterized by a reorganization of the cytoskeletal actin via a process dependent on integrins and the Rho family of GTPases ${ }^{253}$, activation of the $\mathrm{Na}^{+}$channels NHE4 (REF. ${ }^{254}$ ), NKCC1 and NKCC2 (REF. ${ }^{255}$ ), and the resulting responses trigger the expression of heat shock proteins to maintain the correct folding of proteins and activation of the hyperosmolarity-sensitive transcription factor TonEBP (also known as NFAT5); under conditions of prolonged hyperosmolarity, such as in the papilla, these responses ultimately result in the accumulation of inert organic osmolytes ${ }^{252}$. 
4 Fig. 6 | Response of the renal endothelium to changes in osmolarity. a |The renal medullary and papillary regions of the kidney are exposed to hyperosmolarity as a consequence of the countercurrent multiplier and exchange mechanisms, which generates and maintains the medullary hyperosmolarity gradient (ranging from $300 \mathrm{mOsm} / \mathrm{kg} \mathrm{H}_{2} \mathrm{O}$ at the corticomedullary junction to up to $1,400 \mathrm{mOsm}$ in the papilla) that drives the process of urine concentration. $\mathbf{b}$ | In response to a rapid increase in osmolarity (for example, following a switch from diuresis to anti-diuresis) endothelial cells (ECs) tend to shrink as a consequence of water loss. This response results in cytoskeletal rearrangements and activation of a regulatory volume increase (RVI) compensatory mechanism, characterized by an accumulation of intracellular $\mathrm{Na}^{+}$and urea followed by osmotic water reabsorption. Moreover, the expression of heat shock proteins is induced to preserve the correct folding of proteins from high levels of denaturing urea (left panel). Prolonged exposure to hyperosmolarity, such as occurs in the papilla or during situations of prolonged dehydration, induces ECs to promote the production of ATP from oxidative phosphorylation (OXPHOS), and stimulate active $\mathrm{Na}^{+}$ export through the $\mathrm{Na}^{+} / \mathrm{K}^{+}$ATPase as well as the import and synthesis of inert organic osmolytes (such as glucose-derived polyols) to protect the cell from hyperosmolarityinduced cell damage (right panel). AVR, ascending vasa recta; DVR, descending vasa recta; GLUT, glucose transporter; ROS, reactive oxygen species.

Evidence from a transcriptome study in mice exposed to water deprivation suggests that a similar process occurs in the renal medullary endothelium ${ }^{10}$ (FIG. 6b). These mRECs demonstrate increased expression of genes related to glycolytic and oxidative phosphorylation pathways ${ }^{10}$ (FIG. 6b). Moreover, transcriptome analyses have suggested that these cells engage enzymatic and transport processes to aid the accumulation of inert organic osmolytes - such as the sugar derivatives, polyols (in particular, sorbitol, which is derived from the polyol pathway and myo-inositol, which can be taken up or synthesized from glycolytic intermediates), amino acids (in particular, taurine) and possibly methylamines such as betaine and glycerophosphocholine to balance the osmolarity between the intracellular and extracellular compartments ${ }^{10}$ (FIG. 6b). Upon prolonged water deprivation, mRECs also upregulate the $\mathrm{Na}^{+} / \mathrm{K}^{+}$ ATPase, presumably to limit $\mathrm{Na}^{+}$intracellular accumulation (FIG. 6b). In this setting, oxidative phosphorylation, which yields 32 ATP molecules per glucose molecule, would represent a more efficient strategy than anaerobic glycolysis, which releases only two ATP molecules per glucose molecule, to sustain a higher $\mathrm{Na}^{+} / \mathrm{K}^{+}$ATPase activity. Furthermore, some of the glycolysis intermediates that accumulate in ECs exposed to hyperosmolar conditions are likely to deviate from their glycolytic fate to enter osmolyte synthesis pathways, in particular the polyols, sorbitol and myo-inositol ${ }^{10}$. Together, these metabolic adaptations of mRECs to changes in osmolarity would enable their survival in extreme hyperosmolar environments and enable them to sustain kidney function $^{10,256}$.

Regulatory volume increase (RVI). A cellular process that occurs in response to cell shrinkage due to intracellular water loss upon exposure to hyperosmolar conditions. It consists of a compensatory entry of diffusible ions and small metabolites, followed by osmotically driven water influx to restore cell volume.
RNA-seq studies on RECs, is the presence of some typical angiogenic ECs in the kidney cortex and medulla in healthy adult mice ${ }^{6,10}$. These RECs are characterized by a genetic programme resembling that of angiogenic migratory ECs (also known as tip cells) (FIG. 7a). These cells are probably exposed to angiogenic growth factors and may contribute to vascular regeneration in the adult vasculature, although their role is still unknown. Cells within the kidney can produce angiogenic growth factors, either in a constitutive manner (for example, in the case of podocyte-derived VEGFA) or in response to changes in microenvironmental conditions such as hypoxia, oxidative stress, shear stress or inflammation. Angiogenic growth factors drive vascularization of the glomeruli and tubules during kidney development in a process that mainly depends on the cooperation of sprouting and intussusceptive angiogenesis ${ }^{257}$ (FIG. 7a,b). For instance, angiopoietins and their cognate endothelial receptor Tie2 support lymphangiogenesis and are critical for the development of the AVR in mouse embryos, and are necessary for urine concentration ability ${ }^{30}$. VEGF signalling is also required for the proper development of glomerular architecture. For instance, antibody-mediated neutralization of VEGF in newborn mice impairs vessel formation in the superficial renal cortex and glomeruli, and ultimately impairs nephron development ${ }^{258}$, whereas postnatal deletion of VEGFR2 damages the glomerular endothelium ${ }^{48}$. Moreover, loss of podocyte-derived VEGFA precludes glomerular vascularization and leads to perinatal death, whereas heterozygotic loss of Vegfa results in glomerular endotheliosis and proteinuria ${ }^{49}$. Podocyte-derived VEGF is therefore a critical cue for establishing the glomerular endothelium and maintaining it in adulthood $^{257}$. Similarly, tubular epithelial cell-derived VEGFA is necessary for the development and maintenance of peritubular capillaries ${ }^{50}$.

As with endothelial activation in immune activation, the physiological response of RECs to angiogenic signalling may become dysregulated in disease conditions ${ }^{259}$. For example, dysregulated angiogenesis is observed in rejected kidney allografts ${ }^{260}$. Moreover, the loss of angiogenic factors, mainly VEGF, has been implicated in capillary loss after $\mathrm{AKI}^{261}$; conversely, VEGF upregulation improves kidney function, and VEGF administration reduces capillary rarefaction in the context of ischaemia-reperfusion injury ${ }^{262,263}$. Experimental and clinical evidence suggests that in $\mathrm{CKD}$, the balance between pro-angiogenic and anti-angiogenic factors is disturbed ${ }^{259,264}$. Although CKD is characterized by the rarefaction of peritubular capillaries, which promotes kidney hypoxia, prevents the redifferentiation of regenerating tubules and is likely to be associated with endothelial-to-mesenchymal transition, which contributes to kidney fibrosis, proangiogenic signalling in capillary gRECs favours the disruption of the filtration barrier ${ }^{259}$. For instance, evidence from animal models suggests that in early phases of diabetic kidney disease, the glomerular endothelium and ECs found within the vicinity of the glomerulus undergo VEGF-mediated abnormal angiogenesis; blockade of angiogenesis in these models improves kidney function ${ }^{265}$. 


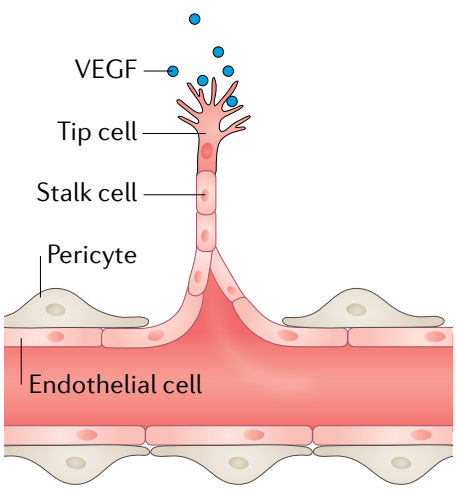

Sprout initiation and extension

b Intussusceptive angiogenesis

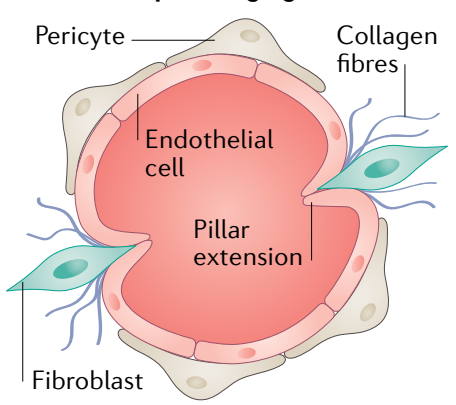

Transcapillary pillar formation

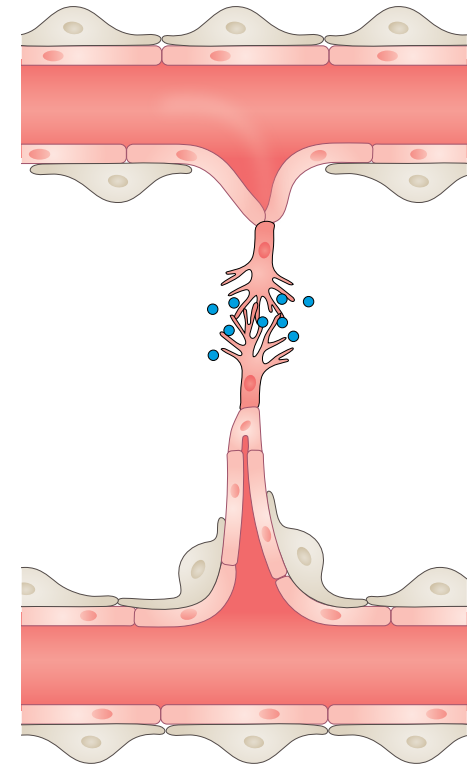

Lumen formation and fusion

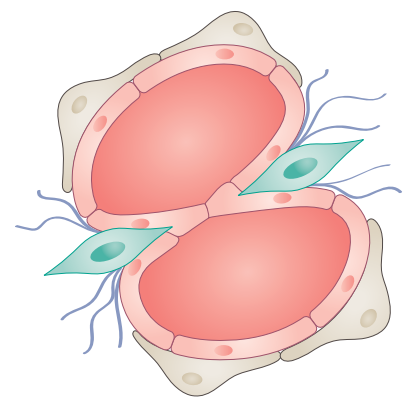

Vascular splitting

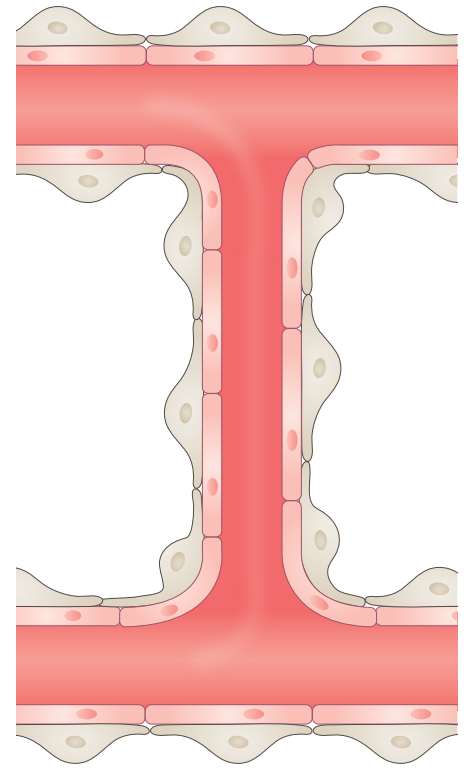

Vessel maturation and stabilization

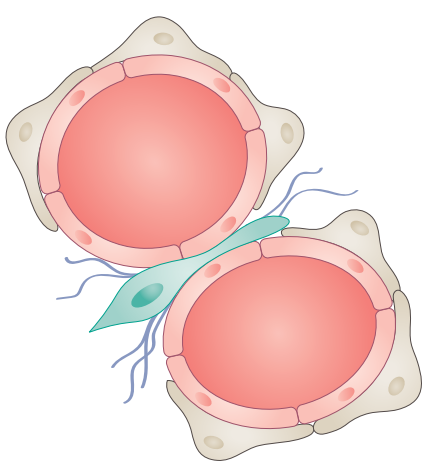

Vessel stabilization

Fig. 7 | Sprouting and intussusceptive angiogenesis. a | Sprouting angiogenesis occurs after a stimulus with an angiogenic growth factor such as VEGF, which activates endothelial cells on pre-existing blood vessels. The activated endothelial cells (ECs), called tip cells, release enzymes that degrade the basement membrane to allow the ECs to migrate from the pre-existing blood vessel, initiating the sprout. The endothelial cells that follow the tip cells (called stalk cells) proliferate to enable extension of the sprout towards the angiogenic stimulus. When two tip cells meet they fuse to form a new capillary lumen that undergoes further vessel maturation and stabilization. $\mathbf{b}$ | Intussusceptive angiogenesis, also called splitting angiogenesis, occurs by splitting a pre-existing blood vessel into two. This process begins with the formation of a pillar extension that protrudes towards the vessel lumen, and forms a transcapillary pillar that splits the vessel into two. Concurrently, myofibroblasts migrate towards the new pillar to help stabilize the newly formed vessels through the deposition of collagen fibres.

While little is known about intussusceptive vessel growth, sprouting angiogenesis has been intensively characterized $^{1}$. The sprouting process is orchestrated through the activation of EC surface receptors and downstream signalling pathways by angiogenic growth factors, and the emergence of two major angiogenic EC phenotypes - proliferating ECs (also known as stalk cells) and migratory ECs (also known as tip cells; described in detail elsewhere $\left.{ }^{1,266,267}\right)$. At the metabolic level, each angiogenic EC type has specific needs (energetic and biomass demands), according to their proliferative or migratory phenotypes, and adapt their metabolism accordingly ${ }^{144}$ (BOX 1). Whether angiogenic RECs, identified by single-cell RNA-seq studies in healthy adult mice exhibit phenotypic and metabolic activity similar to that of tip cells remains to be determined. Moreover, the potential ability of metabolic strategies such as metabolic supplementation to promote angiogenesis or lymphangiogenesis for kidney repair and regeneration also requires further investigation.

\section{Angiocrine signalling in kidney}

ECs also directly instruct their neighbouring cells through the release of growth factors and cytokines, and the expression of adhesion molecules ${ }^{268}$. In the kidney, the vasculature establishes a niche that sustains the development and maintenance of the kidney structure and function ${ }^{4,11}$. Direct proof for this concept is provided by studies of induced pluripotent stem cell-derived kidney organoids, in which maturation of glomerular and 
tubular structures can be induced by promoting vascularization and blood flow through transplantation of the organoid under the kidney capsule of mice ${ }^{269}$. In addition, disruption of the glomerular endothelium in embryonic mouse kidneys prevents podocyte maturation, in particular, the acquisition of foot processes and slit diaphragms ${ }^{49}$. Interestingly, both the developing and adult kidney vasculature demonstrate spatial differences in the expression of angiocrine factors. During the development of the glomerulus, EC-derived matrix metalloproteinase 2 seems to regulate the interaction of ECs with podocytes for glomerular assembly ${ }^{270}$, whereas expression of PDGFB stimulates the recruitment of mesangial cells ${ }^{271,272}$. The developing glomerular endothelium also expresses growth factor genes such as Fgfl and Vegfa, the Notch ligand Dll4, the neuronal guidance cue Sema $5 a$ and the Wnt antagonist Dkk2 (REFS ${ }^{4,11}$ ); $D k k 2$ is also enriched in afferent arteriole gRECs that are associated with the JGA in adulthood ${ }^{10}$. The developing renal arteries and arterioles also express growth factors, including Pdgfb, Tgfb2 and Ltbp4 (REF. ${ }^{11}$ ); expression of $T g f b 2$ and $L t b p 4$ remain enriched in the same REC subsets in adulthood ${ }^{10}$. Strikingly, the insulin-like growth factor signalling system shows a clear zonation within the adult kidney vasculature: $I g f 1$ is mainly expressed in the kidney medulla by RECs of the AVR in the papilla, $I g f b p 7$ is considered a broad marker of mRECs, Igfbp 4 is found in the afferent arteriolar gRECs, Igfbp 3 is mostly expressed by peritubular capillary cRECs and $\operatorname{Ig} f b p 5$ is enriched in both cortical peritubular and glomerular capillaries $^{10,11}$. Multiple other angiocrine factors also show heterogeneous expression among the different EC

\section{Box 1 | Endothelial cell metabolism in sprouting angiogenesis}

Exposure of endothelial cells (ECs) to growth factors, such as VEGF and fibroblast growth factor (FGF), induces the upregulation of glycolytic enzymes such as hexokinase 2 (HK2) and PFKFB3, and overall glycolytic flux, which promotes the differentiation of endothelial tip cells that are responsible for initiation of the sprouting process and migration ${ }^{163,285,286}$. Under conditions of low oxygen and low glucose a situation that tip ECs often encounter - internal glycogen reservoirs can also be mobilized to sustain EC survival and migration ${ }^{287}$. In addition to glucose, glutamine is also necessary for the production of amino acids, such as proline (which is further utilized for collagen biosynthesis), for anaplerosis (that is, to replenish tricarboxylic acid (TCA) cycle intermediates) and for glutathione synthesis to maintain redox homeostasis ${ }^{143,288,289}$. Tip ECs also synthesize fatty acids, which are phospholipid precursors, to maintain optimal membrane fluidity, which is necessary for cell motility ${ }^{290,291}$. For the same purpose, cholesterol export is tightly regulated ${ }^{292}$.

By contrast, proliferative stalk cells mainly use energy for biomass production and for this purpose they show an increase in glycolytic flux in perinuclear locations, through upregulation of PFKFB3 (REF. ${ }^{163}$ ). In addition to ATP, glycolysis yields pyruvate, which is metabolized to acetyl-CoA to enter the TCA cycle, which in turn provides precursors for the synthesis of ribonucleoside triphosphate (rNTP), deoxynucleoside triphosphate (dNTP) and amino acids. Accordingly, proliferative ECs upregulate glycolytic and TCA cycle genes as well as genes involved in nucleotide synthesis ${ }^{143}$. Moreover, both oxidative and non-oxidative pentose phosphate pathways, which are side pathways of glycolysis, are also upregulated to provide ribose, which is necessary for nucleotide synthesis ${ }^{287}$. In addition to glucose catabolism, glutamine breakdown occurs in proliferative ECs, especially to replenish components of the TCA cycle, providing carbons and nitrogen for amino acid and nucleotide synthesis ${ }^{288,293}$. Unlike most other cell types, proliferative stalk ECs rely on fatty acid oxidation for nucleotide and amino acid synthesis, not by replenishing the TCA cycle via anaplerosis, but by sustaining the TCA cycle in conjunction with anaplerotic substrates ${ }^{278,280}$. The metabolic heterogeneity of quiescent and angiogenic ECs has been described in detail elsewhere ${ }^{144}$. phenotypes in adult mouse kidneys ${ }^{10}$. Their roles in the maintenance of kidney tissue and functions remain to be investigated.

Angiocrine signalling is also critical for the repair processes after kidney injury. For instance, RECs may contribute to renal recovery after AKI by regulating inflammatory responses or providing pro-tubulogenic signals $^{273}$. In cocultures, renal proximal tubule cells stimulate RECs to express a number of angiocrine factors, including VEGF, TGF $\beta$, its antagonist $\alpha 2$-macroglobulin and hepatocyte growth factor, which in turn promote the survival, proliferation and differentiation of the proximal tubule cell $\mathrm{s}^{274}$. It is likely that capillary rarefaction induces 'chronic injury-dysregulated repair' processes, which result in impaired epithelial regeneration, nephron loss, and pro-inflammatory and fibrotic signals, possibly due to insufficient angiocrine signals ${ }^{273}$. In support of this hypothesis is evidence demonstrating that endothelial progenitor cell extracts possess renoprotective activity in animal models of AKI by preventing capillary loss and reducing fibrosis, with angiocrine factors such as leukaemia inhibitory factor preventing and reversing fibroblast-to-myofibroblast transition $^{275}$.

Further investigation of the physiological and pathological microenvironments that shape the angiocrine profiles of RECs is warranted. Moreover, the mechanisms by which REC metabolic processes interlink with these distinct angiocrine signatures remain to be determined. Interestingly, a 2020 study described angiocrine metabolite communication in muscle regeneration after ischaemia. That study demonstrated that EC-derived lactate induces macrophages to adopt a pro-regenerative M2-like phenotype, raising the question of whether a similar mechanism might occur in the kidney to promote regeneration after ischaemic insults ${ }^{276}$.

\section{Conclusions}

The mammalian vascular system has long been considered to be a passive conduit for the delivery of oxygen and nutrients to the different organs, as well as for waste removal, with the endothelium serving as blood-tissue barrier. This paradigm is no longer valid since intensive research in the vascular field has revealed an active role for the endothelium not only in the regulation of vasotone and inflammation, but also in organ maturation through the production of angiocrine mediators, immunity and organ function. The importance of ECs to these processes is evidenced by the fact that each organ benefits from a unique endothelium, tailored to its specific needs, and shaped by the cellular and non-cellular microenvironment.

Endothelium in the kidney is unique to that organ and exhibits vast heterogeneity to sustain the multiple activities of the kidney in fluid and blood pressure homeostasis. Importantly, REC dysfunction is not only associated with kidney disease but can drive disease progression, identifying the kidney endothelium as a relevant therapeutic target. In the past decade, extensive analyses have revealed that the metabolism of ECs is tightly regulated at the cellular and subcellular levels, and that different EC phenotypes display unique 
metabolic profiles. The plasticity of EC metabolism provides a mechanism to orchestrate EC phenotypic behaviour, enabling ECs to actively respond to changes in their microenvironment, but also providing a potential opportunity for therapeutic targeting. For example, inhibition of endothelial PFKFB3 in tumour ECs normalized glycolytic flux and improved vessel maturation associated with decreased endothelial activation and tightening of the endothelial barrier ${ }^{277}$. Moreover, supplementation of ECs with the acetyl-CoA precursor, acetate, promotes (lymph)angiogenesis, in vitro and in vivo ${ }^{278-280}$. Specific diets may also offer new therapeutic avenues: a 2019 study demonstrated that a ketogenic diet promotes lymphangiogenesis by increasing the pool of acetyl-CoA in LECs, and decreases lymphoedema in an animal model ${ }^{281}$.

Similarly, in the kidney, the phenotypic heterogeneity of RECs is thought to be driven by metabolic adaptations to cellular needs and signals from the microenvironment in both health and disease. Metabolic targeting of the endothelial dysfunction as well as angiogenesis and lymphangiogenesis are potential strategies to improve kidney function in the context of kidney disease. Kidney organoids have been proposed as a regenerative approach to replace kidney function but their lack of developed vasculature is a major limitation ${ }^{282-284}$. Strategies (including metabolic strategies) to stimulate kidney organoid vascularization and promote EC maturation may promote the maturation of these organoids and ultimately facilitate their therapeutic use ${ }^{269}$. Despite the need for further investigation into the specificities of the renal endothelium, available evidence supports a key role for these cells in kidney physiological processes and suggests that (metabolic) targeting of RECs could be a highly beneficial strategy for promoting renal recovery and regeneration.

Published online 25 March 2021
1. Carmeliet, P. \& Jain, R. K. Molecular mechanisms and clinical applications of angiogenesis. Nature $\mathbf{4 7 3}$ 298-307 (2011)

2. Wong, B. W., Zecchin, A., Garcia-Caballero, M. \& Carmeliet, P. Emerging concepts in organ-specific lymphatic vessels and metabolic regulation of lymphatic development. Dev. Cell 45, 289-301 (2018).

3. Marcu, R. et al. Human organ-specific endothelial cell heterogeneity. iScience 4, 20-35 (2018).

4. Nolan, D. J. et al. Molecular signatures of tissuespecific microvascular endothelial cell heterogeneity in organ maintenance and regeneration. Dev. Cell 26 , 204-219 (2013).

5. Augustin, H. G. \& Koh, G. Y. Organotypic vasculature: from descriptive heterogeneity to functional pathophysiology. Science 357, eaal2379 (2017).

6. Kalucka, J. et al. Single-cell transcriptome atlas of murine endothelial cells. Cell 180, 764-779.e20 (2020).

7. Herzlinger, D. $\&$ Hurtado, R. Patterning the renal vascular bed. Semin. Cell Dev. Biol. 36, 50-56 (2014).

8. Jourde-Chiche, N. et al. Endothelium structure and function in kidney health and disease. Nat. Rev. Nephrol. 15, 87-108 (2019)

9. Molema, G. \& Aird, W. C. Vascular heterogeneity in the kidney. Semin. Nephrol. 32, 145-155 (2012).

10. Dumas, S. J. et al. Single-cell RNA sequencing reveals renal endothelium heterogeneity and metabolic adaptation to water deprivation. J. Am. Soc. Nephrol. 31, 118-138 (2020).

11. Barry, D. M. et al. Molecular determinants of nephron vascular specialization in the kidney. Nat. Commun. 10, 5705 (2019)

12. Jang, C. et al. Metabolite exchange between mammalian organs quantified in pigs. Cell Metab. 30, 594-606.e3 (2019).

13. Wong, B. W., Marsch, E., Treps, L., Baes, M. \& Carmeliet, P. Endothelial cell metabolism in health and disease: impact of hypoxia. EMBO J. 36, 2187-2203 (2017).

14. Makris, K. \& Spanou, L. Acute kidney injury: definition, pathophysiology and clinical phenotypes. Clin. Biochem. Rev. 37, 85-98 (2016).

15. Levey, A. S. et al. Definition and classification of chronic kidney disease: a position statement from Kidney Disease: Improving Global Outcomes (KDIGO). Kidney Int 67, 2089-2100 (2005).

16. Verma, S. K. \& Molitoris, B. A. Renal endothelial injury and microvascular dysfunction in acute kidney injury. Semin. Nephrol. 35, 96-107 (2015).

17. Goligorsky, M. S. Pathogenesis of endothelial cell dysfunction in chronic kidney disease: a retrospective and what the future may hold. Kidney Res. Clin. Pract. 34, 76-82 (2015).

18. Rosivall, L. \& Peti-Peterdi, J. Heterogeneity of the afferent arteriole-correlations between morphology and function. Nephrol. Dial. Transpl. 21, 2703-2707 (2006).

19. Guerci, P., Ergin, B. \& Ince, C. The macro- and microcirculation of the kidney. Best Pract. Res. Clin. Anaesthesiol. 31, 315-329 (2017).
20. Wang, K. \& Kestenbaum, B. Proximal tubular secretory clearance: a neglected partner of kidney function. Clin. J. Am. Soc. Nephrol. 13, 1291-1296 (2018).

21. Ogobuiro, I. Wehrle, C. J., \& Tuma, F. in StatPearls (StatPearls Publishing, 2020).

22. Bobulescu, I. A. \& Moe, O. W. $\mathrm{Na}+/ \mathrm{H}+$ exchangers in renal regulation of acid-base balance. Semin. Nephrol. 26, 334-344 (2006)

23. Stanton, B. A. \& Giebisch, G. H. Potassium transport by the renal distal tubule: effects of potassium loading. Am. J. Physiol. 243, F487-F493 (1982).

24. Eisner, $C$. et al. Major contribution of tubular secretion to creatinine clearance in mice. Kidney Int. 77 , 519-526 (2010)

25. Berkhin, E. B. \& Humphreys, M. H. Regulation of renal tubular secretion of organic compounds. Kidney Int. 59, 17-30 (2001)

26. Pannabecker, T. L. \& Layton, A. T. Targeted delivery of solutes and oxygen in the renal medulla: role of microvessel architecture. Am. J. Physiol. Ren. Physiol. 307, F649-F655 (2014)

27. O'Morchoe, C. C. \& O'Morchoe, P. J. The renal lymphatic system: a brief review. Contrib. Nephrol. 68, 230-237 (1988)

28. Russell, P. S., Hong, J., Windsor, J. A., Itkin, M ¿ Phillips, A. R. J. Renal lymphatics: anatomy, physiology, and clinical implications. Front. Physiol. 10, 251 (2019).

29. Seeger, H., Bonani, M. \& Segerer, S. The role of lymphatics in renal inflammation. Nephrol. Dial. Transpl. 27, 2634-2641 (2012)

30. Kenig-Kozlovsky, Y. et al. Ascending vasa recta are angiopoietin/Tie2-dependent lymphatic-like vessels. J. Am. Soc. Nephrol. 29, 1097-1107 (2018).

31. Ribatti, D., Nico, B., Vacca, A., Roncali, L. \& Dammacco, F. Endothelial cell heterogeneity and organ specificity. J. Hematother. Stem Cell Res. 11, 81-90 (2002).

32. Brunskill, E. W. \& Potter, S. S. Gene expression programs of mouse endothelial cells in kidney development and disease. PLOS ONE 5, e 12034 (2010).

33. Huang, T. W. Basal lamina heterogeneity in the glomerular capillary tufts of human kidneys. J. Exp. Med. 149, 1450-1459 (1979).

34. Dantzler, W. H., Layton, A. T., Layton, H. E. \& Pannabecker, T. L. Urine-concentrating mechanism in the inner medulla: function of the thin limbs of the loops of Henle. Clin. J. Am. Soc. Nephrol. 9 1781-1789 (2014).

35. Sorensen, C. M. et al. Role of connexin 40 in the autoregulatory response of the afferent arteriole. Am. J. Physiol Ren Physiol 303, F855-F863 (2012).

36. Burke, M., Pabbidi, M. R., Farley, J. \& Roman, R. J. Molecular mechanisms of renal blood flow autoregulation. Curr. Vasc. Pharmacol. 12, 845-858 (2014).

37. Barajas, L. Anatomy of the juxtaglomerular apparatus. Am. J. Physiol. 237, F333-F343 (1979).

38. Satchell, S. C. \& Braet, F. Glomerular endothelial cell fenestrations: an integral component of the glomerular filtration barrier. Am. J. Physiol. Ren. Physiol. 296, F947-F956 (2009).

39. Jeansson, M. \& Haraldsson, B. Morphological and functional evidence for an important role of the endothelial cell glycocalyx in the glomerular barrier Am. J. Physiol. Ren. Physiol. 290, F111-F116 (2006).

40. van den Berg, B. M. et al. Glomerular function and structural integrity depend on hyaluronan synthesis by glomerular endothelium. J. Am. Soc. Nephrol. 30, 1886-1897 (2019).

41. Hjalmarsson, C., Johansson, B. R. \& Haraldsson, B. Electron microscopic evaluation of the endothelial surface layer of glomerular capillaries. Microvasc. Res. 67, 9-17 (2004).

42. Scott, R. P. \& Quaggin, S. E. Review series: the cell biology of renal filtration. J. Cell Biol. 209, 199-210 (2015).

43. Friden, V. et al. The glomerular endothelial cell coat is essential for glomerular filtration. Kidney Int. 79, 1322-1330 (2011).

44. Dane, M. J. et al. Glomerular endothelial surface layer acts as a barrier against albumin filtration. Am. J. Pathol. 182, 1532-1540 (2013).

45. Hudson, B. G., Tryggvason, K., Sundaramoorthy, M $\&$ Neilson, E. G. Alport's syndrome, Goodpasture's syndrome, and type IV collagen. N. Engl. J. Med. 348 2543-2556 (2003).

46. Zenker, $\mathrm{M}$. et al. Human laminin $\beta 2$ deficiency causes congenital nephrosis with mesangial sclerosis and distinct eye abnormalities. Hum. Mol. Genet. 13, 2625-2632 (2004).

47. Stan, R. V., Kubitza, M. \& Palade, G. E. PV-1 is a component of the fenestral and stomatal diaphragms in fenestrated endothelia. Proc. Natl Acad. Sci. USA 96, 13203-13207 (1999).

48. Sison, K. et al. Glomerular structure and function require paracrine, not autocrine, VEGF-VEGFR-2 signaling. J. Am. Soc. Nephrol. 21, 1691-1701 (2010).

49. Eremina, V. et al. Glomerular-specific alterations of VEGF-A expression lead to distinct congenital and acquired renal diseases. J. Clin. Invest. 111, 707-716 (2003).

50. Dimke, H. et al. Tubulovascular cross-talk by vascular endothelial growth factor A maintains peritubular microvasculature in kidney. J. Am. Soc. Nephrol. 26, 1027-1038 (2015).

51. Kriz, W. \& Lemley, K. V. Potential relevance of shear stress for slit diaphragm and podocyte function. Kidney Int. 91, 1283-1286 (2017).

52. Hazell, G. G. et al. PI16 is a shear stress and inflammation-regulated inhibitor of MMP2. Sci. Rep. 6, 39553 (2016).

53. Patrakka, J. et al. Expression and subcellular distribution of novel glomerulus-associated proteins dendrin, ehd3, sh2d4a, plekhh2, and 2310066E14Rik. J. Am. Soc. Nephrol. 18, 689-697 (2007).

54. George, M. et al. Renal thrombotic microangiopathy in mice with combined deletion of endocytic recycling regulators EHD3 and EHD4. PLOS ONE 6, e 17838 (2011). 
55. Stewart, B. J. et al. Spatiotemporal immune zonation of the human kidney. Science 365, 1461-1466 (2019).

56. Cai, J., Pardali, E., Sanchez-Duffhues, G. \& ten Dijke, P. BMP signaling in vascular diseases. FEBS Lett. 586, 1993-2002 (2012).

57. Van Themsche, C., Chaudhry, P., Leblanc, V., Parent, S. $\&$ Asselin, E. XIAP gene expression and function is regulated by autocrine and paracrine TCF- $\beta$ signaling. Mol. Cancer 9, 216 (2010).

58. Shang, Y. et al. Transcriptional corepressors HIPK and HIPK2 control angiogenesis via TGF- $\beta$-TAK 1 dependent mechanism. PLoS Biol. 11, e 1001527 (2013).

59. Ghayur, A. \& Margetts, P. J. Transforming growth factor-beta and the glomerular filtration barrier. Kidney Res. Clin. Pract. 32, 3-10 (2013).

60. Ueda, H. et al. Bmp in podocytes is essential for normal glomerular capillary formation. J. Am. Soc Nephrol. 19, 685-694 (2008).

61. Herman-Edelstein, M., Scherzer, P., Tobar, A., Levi, M. \& Gafter, U. Altered renal lipid metabolism and renal lipid accumulation in human diabetic nephropathy. J. Lipid Res. 55, 561-572 (2014).

62. Kang, H. M. et al. Defective fatty acid oxidation in renal tubular epithelial cells has a key role in kidney fibrosis development. Nat. Med. 21, 37-46 (2015).

63. Corada, M. et al. Sox 17 is indispensable for acquisition and maintenance of arterial identity. Nat. Commun. 4, 2609 (2013)

64. You, L. R. et al. Suppression of Notch signalling by the COUP-TFII transcription factor regulates vein identity. Nature 435, 98-104 (2005)

65. Messaoudi, S. et al. Endothelial Gata5 transcription factor regulates blood pressure. Nat. Commun. $\mathbf{6}$, 8835 (2015).

66. Dalal, R., Bruss, Z. S. \& Sehdev, J. S. in StatPearls (StatPearls Publishing, 2020).

67. Gattone, V. H. 2nd, Luft, F. C. \& Evan, A. P. Renal afferent and efferent arterioles of the rabbit. Am. J. Physiol. 247, F219-F228 (1984).

68. Rosivall, L., Razga, Z \& Ormos, J. Morphological characterization of human juxtaglomerular apparatus. Kidney Int. Suppl. 32, S9-S12 (1991).

69. Rosivall, L. \& Taugner, R. The morphological basis of fluid balance in the interstitium of the juxtaglomerular apparatus. Cell Tissue Res. 243, 525-533 (1986).

70. Haefliger, J. A. et al. Connexins 40 and 43 are differentially regulated within the kidneys of rats with renovascular hypertension. Kidney Int. 60, 190-201 (2001).

71. Just, A. et al. Connexin 40 mediates the tubuloglomerular feedback contribution to renal blood flow autoregulation. J. Am. Soc. Nephrol. 20, 1577-1585 (2009)

72. Guan, Z., VanBeusecum, J. P. \& Inscho, E. W. Endothelin and the renal microcirculation. Semin. Nephrol. 35, 145-155 (2015).

73. Dobrian, A. D. et al. Functional and pathological roles of the 12- and 15-lipoxygenases. Prog. Lipid Res. 50, 115-131 (2011).

74. Igarashi, J. \& Michel, T. Sphingosine-1-phosphate and modulation of vascular tone. Cardiovasc. Res. 82 212-220 (2009).

75. Guan, Z. et al. Sphingosine-1-phosphate evokes unique segment-specific vasoconstriction of the renal microvasculature. J. Am. Soc. Nephrol. 25, 1774-1785 (2014)

76. Guan, Z., Wang, F., Cui, X. \& Inscho, E. W. Mechanisms of sphingosine-1-phosphate-mediated vasoconstriction of rat afferent arterioles. Acta Physiol. 222, e12913 (2018).

77. Zhang, J. \& Hill, C. E. Differential connexin expression in preglomerular and postglomerular vasculature: accentuation during diabetes. Kidney Int. 68 1171-1185 (2005)

78. Wright, E. M., Hirayama, B. A. \& Loo, D. F. Active sugar transport in health and disease. J. Intern. Med. 261, 32-43 (2007)

79. Mather, A. \& Pollock, C. Glucose handling by the kidney. Kidney Int. Suppl. 79(Suppl. 120), S1-S6 (2011).

80. Busnelli, M., Manzini, S., Parolini, C., EscalanteAlcalde, D. \& Chiesa, G. Lipid phosphate phosphatase 3 in vascular pathophysiology. Atherosclerosis 271 , 156-165 (2018)

81. Yao, D. W. et al. Thyroid hormone responsive (THRSP) promotes the synthesis of medium-chain fatty acids in goat mammary epithelial cells. J. Dairy Sci. 99 3124-3133 (2016)

82. Welti, J., Loges, S., Dimmeler, S. \& Carmeliet, P. Recent molecular discoveries in angiogenesis and antiangiogenic therapies in cancer. J. Clin. Invest. 123 3190-3200 (2013)

83. Pollak, M. The insulin and insulin-like growth factor receptor family in neoplasia: an update. Nat. Rev. Cancer 12, 159-169 (2012).

84. Matsukawa, N. et al. The natriuretic peptide clearance receptor locally modulates the physiological effects of the natriuretic peptide system. Proc. Natl Acad. Sci. USA 96, 7403-7408 (1999).

85. Potter, L. R. Natriuretic peptide metabolism, clearance and degradation. FEBS J. 278, 1808-1817 (2011).

86. Morita, K., Sasaki, H., Furuse, M. \& Tsukita, S. Endothelial claudin: claudin-5/TMVCF constitutes tight junction strands in endothelial cells. J. Cell Biol. 147, 185-194 (1999).

87. Buschmann, I. et al. Pulsatile shear and Gja5 modulate arterial identity and remodeling events during flow-driven arteriogenesis. Development 137, 2187-2196 (2010)

88. Fang, J. S. et al. Shear-induced Notch-Cx37-p27 axis arrests endothelial cell cycle to enable arterial specification. Nat. Commun. 8, 2149 (2017)

89. High, F. A. et al. Endothelial expression of the Notch ligand Jagged 1 is required for vascular smooth muscle development. Proc. Natl Acad. Sci. USA 105 1955-1959 (2008).

90. Kutschera, S et al. Differential endothelial transcriptomics identifies semaphorin $3 \mathrm{G}$ as a vascula class 3 semaphorin. Arterioscler. Thromb. Vasc. Biol. 31, 151-159 (2011).

91. dela Paz, N. G. \& D'Amore, P. A. Arterial versus venous endothelial cells. Cell Tissue Res. 335, 5-16 (2009).

92. Chapman, S. L. et al. Fibulin-2 and fibulin-5 cooperatively function to form the internal elastic lamina and protect from vascular injury. Arterioscler. Thromb. Vasc. Biol. 30, 68-74 (2010).

93. Noda, K. et al. Latent TGF- $\beta$ binding protein 4 promotes elastic fiber assembly by interacting with fibulin-5. Proc. Natl Acad. Sci. USA 110, 2852-2857 (2013).

94. Tojais, N. F. et al. Codependence of bone morphogenetic protein receptor 2 and transforming growth factor- $\beta$ in elastic fiber assembly and its perturbation in pulmonary arterial hypertension. Arterioscler. Thromb. Vasc. Biol. 37, 1559-1569 (2017).

95. Wagenseil, J. E. \& Mecham, R. P. Elastin in large artery stiffness and hypertension. J. Cardiovasc. Transl. Res. 5, 264-273 (2012).

96. Bjorklund, G. et al. The role of matrix Gla protein (MGP) in vascular calcification. Curr. Med. Chem. 27, 1647-1660 (2020).

97. Arendshorst, W. J., Chatziantoniou, C. \& Daniels, F. H. Role of angiotensin in the renal vasoconstriction observed during the development of genetic hypertension. Kidney Int. Suppl. 30, S92-S96 (1990).

98. Cantalupo, A. et al. S1PR1 (sphingosine-1-phosphate receptor 1) signaling regulates blood flow and pressure. Hypertension 70, 426-434 (2017).

99. Masaki, T. Possible role of endothelin in endothelial regulation of vascular tone. Annu. Rev. Pharmacol. Toxicol. 35, 235-255 (1995).

100. Ritman, E. L. \& Lerman, A. The dynamic vasa vasorum. Cardiovasc. Res. 75, 649-658 (2007).

101. Holmes, M. J., O'Morchoe, P. J. \& O'Morchoe, C. C. Morphology of the intrarenal lymphatic system. Capsular and hilar communications. Am. J. Anat. 149 333-351 (1977)

102. Breiteneder-Geleff, S. et al. Podoplanin, novel 43-kd membrane protein of glomerular epithelial cells, is down-regulated in puromycin nephrosis. Am. J. Pathol. 151, 1141-1152 (1997).

103. Banerji, S. et al. LYVE-1, a new homologue of the CD44 glycoprotein, is a lymph-specific receptor for hyaluronan. J. Cell Biol. 144, 789-801 (1999).

104. Jeltsch, M. et al. Hyperplasia of lymphatic vessels in VEGF-C transgenic mice. Science 276, 1423-1425 (1997).

105. Wigle, J. T. \& Oliver, G. Prox 1 function is required for the development of the murine lymphatic system. Cell 98, 769-778 (1999)

106. Kurbel, S., Dodig, K. \& Radic, R. The osmotic gradient in kidney medulla: a retold story. Adv. Physiol. Educ. 26, 278-281 (2002)

107. Aregger, F. et al. Identification of IGFBP-7 by urinary proteomics as a novel prognostic marker in early acut kidney injury. Kidney Int. 85, 909-919 (2014).

108. Jay, A. G. \& Hamilton, J. A. The enigmatic membrane fatty acid transporter CD36: new insights into fatty acid binding and their effects on uptake of oxidized LDL. Prostaglandins Leukot. Essent. Fatty Acids 138, 64-70 (2018).
109. Bohman, S. O. \& Jensen, P. K. Morphometric studies on the lipid droplets of the interstitial cells of the renal medulla in different states of diuresis. J. Ultrastruct. Res. 55, 182-192 (1976)

110. Pravenec, M. et al. Identification of renal Cd36 as a determinant of blood pressure and risk for hypertension. Nat. Genet. 40, 952-954 (2008).

111. Okamura, D. M. et al. CD36 regulates oxidative stress and inflammation in hypercholesterolemic CKD J. Am. Soc. Nephrol. 20, 495-505 (2009).

112. Lake, B. B. et al. A single-nucleus RNA-sequencing pipeline to decipher the molecular anatomy and pathophysiology of human kidneys. Nat. Commun. 10 , 2832 (2019).

113. Poulos, M., Redmond, D., Gutkin, M., Ramalingam, P. $\&$ Butler, J. M. Single-cell characterization of the HSC supportive bone marrow vascular microenvironment [abstract]. Blood 132 (Suppl. 1), 2577 (2018).

114. Yang, B. $\&$ Bankir, L. Urea and urine concentrating ability: new insights from studies in mice. $\mathrm{Am}$. J. Physiol. Ren. Physiol. 288, F881-F896 (2005).

115. King, L. S., Choi, M., Fernandez, P. C., Cartron, J. P. \& Agre, P. Defective urinary concentrating ability due to a complete deficiency of aquaporin-1. N. Engl. J. Med. 345, 175-179 (2001)

116. Noda, Y., Horikawa, S., Katayama, Y. \& Sasaki, S Identification of a multiprotein "motor" complex binding to water channel aquaporin-2. Biochem. Biophys. Res. Commun. 330, 1041-1047 (2005).

117. Neuhofer, W. \& Beck, F. X. Cell survival in the hostile environment of the renal medulla. Annu. Rev. Physiol. 67, 531-555 (2005)

118. Schulze Blasum, B. et al. The kidney-specific expression of genes can be modulated by the extracellular osmolality. FASEB J. 30, 3588-3597 (2016).

119. Fesus, G. et al. Adiponectin is a novel humoral vasodilator. Cardiovasc. Res. 75, 719-727 (2007).

120. Silldorff, E. P., Yang, S. \& Pallone, T. L. Prostaglandin E2 abrogates endothelin-induced vasoconstriction in renal outer medullary descending vasa recta of the rat. J. Clin. Invest. 95, 2734-2740 (1995).

121. Evans, R. G., Eppel, G. A., Anderson, W. P. \& Denton, K. M. Mechanisms underlying the differential control of blood flow in the renal medulla and cortex. J. Hypertens. 22, 1439-1451 (2004).

122. Pannabecker, T. L. \& Dantzler, W. H. Threedimensional architecture of inner medullary vasa recta. Am. J. Physiol. Ren. Physiol. 290, F1355-F1366 (2006)

123. Sheikh-Hamad, D. et al. CD9 antigen mRNA is induced by hypertonicity in two renal epithelial cell lines. Am. J. Physiol. 270, C253-C258 (1996).

124. Izumi, Y., Yang, W., Zhu, J., Burg, M. B. \& Ferraris, J. D. RNA-Seq analysis of high $\mathrm{NaCl}$-induced gene expression. Physiol. Genomics 47, 500-513 (2015).

125. Chen, Y., Fry, B. C. \& Layton, A. T. Modeling glucose metabolism and lactate production in the kidney. Math. Biosci. 289, 116-129 (2017).

126. Eelen, G., de Zeeuw, P., Simons, M. ¿ Carmeliet, P. Endothelial cell metabolism in normal and diseased vasculature. Circ. Res. 116, 1231-1244 (2015).

127. Krishnan, D. et al. Deficiency of carbonic anhydrase II results in a urinary concentrating defect. Front. Physiol. 8, 1108 (2017)

128. Rabelink, T. J. \& Luscher, T. F. Endothelial nitric oxide synthase: host defense enzyme of the endothelium? Arterioscler. Thromb. Vasc. Biol. 26, 267-271 (2006).

129. Liu, L. et al. Essential roles of S-nitrosothiols in vascular homeostasis and endotoxic shock. Cell 116, 617-628 (2004).

130. Cooke, J. P. Rossitch, E. Jr, Andon, N. A Loscalzo, J. $\&$ Dzau, V. J. Flow activates an endothelial potassium channel to release an endogenous nitrovasodilator. J. Clin. Invest. 88, 1663-1671 (1991).

131. Amador-Martinez, I. et al. Reduced endothelial nitric oxide synthase activation contributes to cardiovascular injury during chronic kidney disease progression. Am. J. Physiol. Ren. Physiol. 317 , F275-F285 (2019).

132. Rabelink, T. J. et al. Heparanase: roles in cell survival, extracellular matrix remodelling and the development of kidney disease. Nat. Rev. Nephrol. 13, 201-212 (2017).

133. Wang, G. et al. Shear stress regulation of endothelial glycocalyx structure is determined by glucobiosynthesis. Arterioscler. Thromb. Vasc. Biol. 40, 350-364 (2020)

134. Loeven, M. A. et al. Mutations in complement factor $\mathrm{H}$ impair alternative pathway regulation on mouse glomerular endothelial cells in vitro. J. Biol. Chem. 291, 4974-4981 (2016) 
135. Liptak, P. et al. Peritubular capillary damage in acute humoral rejection: an ultrastructural study on human renal allografts. Am. J. Transpl. 5, 2870-2876 (2005).

136. Kida, Y., Tchao, B. N. \& Yamaguchi, I. Peritubular capillary rarefaction: a new therapeutic target in chronic kidney disease. Pediatr. Nephrol. 29 333-342 (2014)

137. Nadim, M. K. et al. COVID-19-associated acute kidney injury: consensus report of the 25th Acute Disease Quality Initiative (ADQI) workgroup. Nat. Rev. Nephrol. 16, 747-764 (2020).

138. Teuwen, L. A., Geldhof, V., Pasut, A. \& Carmeliet, P. COVID-19: the vasculature unleashed. Nat. Rev. Immunol. 20, 389-391 (2020)

139. Varga, Z. et al. Endothelial cell infection and endotheliitis in COVID-19. Lancet 395, 1417-1418 (2020).

140. Drummond, K. \& Mauer, M., International Diabetic Nephropathy Study Group. The early natural history of nephropathy in type 1 diabetes: II. Early renal structural changes in type 1 diabetes. Diabetes $\mathbf{5 1}$ 1580-1587 (2002).

141. Chavers, B. M., Bilous, R. W., Ellis, E. N., Steffes, M. W. \& Mauer, S. M. Glomerular lesions and urinary albumin excretion in type I diabetes without overt proteinuria. N. Engl. J. Med. 320, 966-970 (1989).

142. Brodsky, S. V. et al. Endothelial dysfunction in ischemic acute renal failure: rescue by transplanted endothelial cells. Am. J. Physiol. Ren. Physiol. 282, F1140-F1149 (2002)

143. Rohlenova, K. et al. Single-cell RNA sequencing maps endothelial metabolic plasticity in pathological angiogenesis. Cell Metab. 31, 862-877.e14 (2020)

144. Dumas, S. J., Garcia-Caballero, M. \& Carmeliet, P. Metabolic signatures of distinct endothelial phenotypes. Trends Endocrinol. Metab. 31, 580-595 (2020).

145. Jambusaria, A. et al. Endothelial heterogeneity across distinct vascular beds during homeostasis and inflammation. eLife 9, e51413 (2020).

146. Kalucka, J. et al. Quiescent endothelial cells upregulate fatty acid $\beta$-oxidation for vasculoprotection via redox homeostasis. Cell Metab. 28, 881-894.e13 (2018).

147. Xiong, J. et al. A metabolic basis for endothelialto-mesenchymal transition. Mol. Cell 69, 689-698.e7 (2018).

148. Brezis, M., Rosen, S., Silva, P. \& Epstein, F. H. Renal ischemia: a new perspective. Kidney Int. 26, 375-383 (1984).

149. Gunaratnam, L. \& Bonventre, J. V. HIF in kidney disease and development. J. Am. Soc. Nephrol. 20 1877-1887 (2009).

150. Rosenberger, C. et al. Up-regulation of HIF in experimental acute renal failure: evidence for a protective transcriptional response to hypoxia. Kidney Int. 67, 531-542 (2005).

151. Fine, L. G. \& Norman, J. T. Chronic hypoxia as a mechanism of progression of chronic kidney diseases: from hypothesis to novel therapeutics. Kidney Int. $\mathbf{7 4}$ 867-872 (2008)

152. Haase, V. H. Mechanisms of hypoxia responses in renal tissue. J. Am. Soc. Nephrol. 24, 537-541 (2013).

153. Faller, D. V. Endothelial cell responses to hypoxic stress. Clin. Exp. Pharmacol. Physiol. 26, 74-84 (1999).

154. Bartoszewski, R. et al. Primary endothelial cell-specific regulation of hypoxia-inducible factor (HIF)-1 and HIF-2 and their target gene expression profiles during hypoxia. FASEB J. 33, 7929-7941 (2019).

155. Kalucka, J. et al. Kidney injury is independent of endothelial HIF-1 a. J. Mol. Med. 93, 891-904 (2015).

156. Kapitsinou, P. P. et al. Endothelial HIF-2 mediates protection and recovery from ischemic kidney injury. J. Clin. Invest. 124, 2396-2409 (2014).

157. Rosenberger, C. et al. Expression of hypoxia-inducible factor- $1 \alpha$ and $-2 \alpha$ in hypoxic and ischemic rat kidneys. J. Am. Soc. Nephrol. 13, 1721-1732 (2002).

158. Tang, N. et al. Loss of HIF-1 $\alpha$ in endothelial cells disrupts a hypoxia-driven VEGF autocrine loop necessary for tumorigenesis. Cancer Cell 6, 485-495 (2004).

159. Takeda, K. \& Fong, G. H. Prolyl hydroxylase domain 2 protein suppresses hypoxia-induced endothelial cell proliferation. Hypertension 49, 178-184 (2007).

160. Tanaka, T. et al. Hypoxia-induced apoptosis in cultured glomerular endothelial cells: involvement of mitochondrial pathways. Kidney Int. 64, 2020-2032 (2003).
161. Wang, L. et al. Podocytes protect glomerular endothelial cells from hypoxic injury via deSUMOylation of HIF-1 a signaling. Int. J. Biochem Cell Biol. 58, 17-27 (2015).

162. Luo, P. L., Wang, Y. J., Yang, Y. Y. \& Yang, J. J. Hypoxia-induced hyperpermeability of rat glomerular endothelial cells involves HIF-2 $\alpha$ mediated changes in the expression of occludin and ZO-1. Braz. J. Med. Biol. Res. 51, e6201 (2018)

163. De Bock, K. et al. Role of PFKFB3-driven glycolysis in vessel sprouting. Cell 154, 651-663 (2013).

164. Koziel, A. \& Jarmuszkiewicz, W. Hypoxia and aerobic metabolism adaptations of human endothelial cells. Pflugers Arch. Eur. J.Physiol. 469, 815-827 (2017).

165. Loike, J. D. et al. Hypoxia induces glucose transporter expression in endothelial cells. Am. J. Physiol. 263 C326-C333 (1992)

166. Nauta, T. D. et al. Identification of HIF-2a-regulated genes that play a role in human microvascular endothelial sprouting during prolonged hypoxia in vitro. Angiogenesis 20, 39-54 (2017).

167. He, X. et al. Endothelial specific SIRT3 deletion impairs glycolysis and angiogenesis and causes diastolic dysfunction. J. Mol. Cell Cardiol. 112, 104-113 (2017)

168. Tseng, A. H., Wu, L. H., Shieh, S. S. \& Wang, D. L. SIRT3 interactions with FOXO3 acetylation, phosphorylation and ubiquitinylation mediate endothelial cell responses to hypoxia. Biochem. $J$. 464, 157-168 (2014).

169. Zhang, S. et al. FoxO3a modulates hypoxia stress induced oxidative stress and apoptosis in cardiac microvascular endothelial cells. PLOS ONE 8, e80342 (2013).

170. Lin, J. R. et al. Suppression of endothelialto-mesenchymal transition by SIRT (sirtuin) 3 alleviated the development of hypertensive renal injury. Hypertension 72, 350-360 (2018).

171. Morigi, M. et al. Sirtuin 3-dependent mitochondrial dynamic improvements protect against acute kidney injury. J. Clin. Invest. 125, 715-726 (2015).

172. Singh, N., Singh, H., Jagavelu, K., Wahajuddin, M. \& Hanif, K. Fatty acid synthase modulates proliferation, metabolic functions and angiogenesis in hypoxic pulmonary artery endothelial cells. Eur. J. Pharmacol. 815, 462-469 (2017).

173. Szolkiewicz, M. et al. Upregulation of fatty acid synthase gene expression in experimental chronic renal failure. Metabolism 51, 1605-1610 (2002)

174. Saraf, S. L. et al. Progressive glomerular and tubular damage in sickle cell trait and sickle cell anemia mouse models. Transl. Res. 197, 1-11 (2018).

175. Wahl, P., Ducasa, G. M. \& Fornoni, A. Systemic and renal lipids in kidney disease development and progression. Am. J. Physiol. Ren. Physiol. 310 F433-F445 (2016).

176. Krotova, K., Patel, J. M., Block, E. R. \& Zharikov, S. Hypoxic upregulation of arginase II in human lung endothelial cells. Am. J. Physiol. Cell Physiol. 299, C1541-C1548 (2010).

177. Liang, X., Arullampalam, P., Yang, Z. \& Ming, X. F. Hypoxia enhances endothelial intercellular adhesion molecule 1 protein level through upregulation of arginase type II and mitochondrial oxidative stress. Front. Physiol. 10, 1003 (2019).

178. Block, E. R., Herrera, H. \& Couch, M. Hypoxia inhibits L-arginine uptake by pulmonary artery endothelial cells. Am. J. Physiol. 269, L574-L580 (1995).

179. Su, Y \& Block, E. R. Hypoxia inhibits L-arginine synthesis from L-citrulline in porcine pulmonary artery endothelial cells. Am. J. Physiol. 269, L581-L587 (1995).

180. Goligorsky, M. S., Chen, J. \& Brodsky, S. Workshop: endothelial cell dysfunction leading to diabetic nephropathy: focus on nitric oxide. Hypertension 37 , 744-748 (2001)

181. Huang, J. et al. Genetic targeting of arginase-II in mouse prevents renal oxidative stress and inflammation in diet-induced obesity. Front. Physiol. 7, 560 (2016)

182. Woodward, H. N. et al. PI3K, Rho, and ROCK play a key role in hypoxia-induced ATP release and ATPstimulated angiogenic responses in pulmonary artery vasa vasorum endothelial cells. Am. J. Physiol. Lung Cell Mol. Physiol. 297, L954-L964 (2009).

183. Lim To, W. K., Kumar, P. \& Marshall, J. M. Hypoxia is an effective stimulus for vesicular release of ATP from human umbilical vein endothelial cells. Placenta 36 , 759-766 (2015)

184. Dwyer, K. M., Kishore, B. K. \& Robson, S. C. Conversion of extracellular ATP into adenosine: a master switch in renal health and disease. Nat. Rev. Nephrol 16, 509-524 (2020).
185. Burnstock, G. \& Ralevic, V. Purinergic signaling and blood vessels in health and disease. Pharmacol. Rev. 66, 102-192 (2014).

186. Ledoux, S. et al. Hypoxia enhances Ecto-5'nucleotidase activity and cell surface expression in endothelial cells: role of membrane lipids. Circ. Res. 92, 848-855 (2003).

187. Liu, Z. et al. Endothelial adenosine A2a receptormediated glycolysis is essential for pathological retinal angiogenesis. Nat. Commun. 8, 584 (2017).

188. Ahmad, A. et al. Adenosine A2A receptor is a unique angiogenic target of HIF-2 $\alpha$ in pulmonary endothelial cells. Proc. Natl Acad. Sci. USA 106, 10684-10689 (2009).

189. Bauerle, J. D., Grenz, A., Kim, J. H., Lee, H. T. \& Eltzschig, H. K. Adenosine generation and signaling during acute kidney injury. J. Am. Soc. Nephrol. 22 14-20 (2011)

190. Inscho, E. W. ATP, P2 receptors and the renal microcirculation. Purinergic Signal. 5, 447-460 (2009).

191. Crawford, C et al. Extracellular nucleotides affect pericyte-mediated regulation of rat in situ vasa recta diameter. Acta Physiol. 202, 241-251 (2011).

192. Silldorff, E. P. \& Pallone, T. L. Adenosine signaling in outer medullary descending vasa recta. $A m$. $J$. Physiol. Regul. Integr. Comp. Physiol. 280, R854-R861 (2001).

193. Jackson, E. K., Zhu, C. \& Tofovic, S. P. Expression of adenosine receptors in the preglomerular microcirculation. Am. J. Physiol. Ren. Physiol. 283 F41-F51 (2002)

194. Solini, A., Usuelli, V. \& Fiorina, P. The dark side of extracellular ATP in kidney diseases. J. Am. Soc. Nephrol. 26, 1007-1016 (2015).

195. Baeyens, N., Bandyopadhyay, C., Coon, B. G., Yun, S. \& Schwartz, M. A. Endothelial fluid shear stress sensing in vascular health and disease. J. Clin. Invest. 126, 821-828 (2016)

196. Sugimori, H. et al. Increased blood viscosity is associated with reduced renal function and elevated urinary albumin excretion in essential hypertensives without chronic kidney disease. Hypertens. Res. 36 247-251 (2013)

197. Ballermann, B. J., Dardik, A., Eng, E. \& Liu, A. Shear stress and the endothelium. Kidney Int. Suppl. 67, S100-S108 (1998).

198. Tati, R. et al. Phenotypic expression of ADAMTS 13 in glomerular endothelial cells. PLOS ONE 6, e21587 (2011).

199. Dong, J. F. et al. ADAMTS-13 rapidly cleaves newly secreted ultralarge von Willebrand factor multimers on the endothelial surface under flowing conditions. Blood 100, 4033-4039 (2002).

200. Nolasco, L. H. et al. Hemolytic uremic syndromeassociated Shiga toxins promote endothelial-cell secretion and impair ADAMTS13 cleavage of unusually large von Willebrand factor multimers. Blood 106, 4199-4209 (2005).

201. Malek, A. M., Alper, S. L. \& Izumo, S. Hemodynamic shear stress and its role in atherosclerosis. JAMA $\mathbf{2 8 2}$ 2035-2042 (1999).

202. Tafur-Soto, J. D. \& White, C. J. Renal artery stenosis. Cardiol. Clin. 33, 59-73 (2015).

203. Sathanoori, R. et al. Shear stress modulates endothelial KLF2 through activation of P2X4. Purinergic Signal. 11, 139-153 (2015).

204. Slater, S. C. et al. Chronic exposure to laminar shea stress induces Kruppel-like factor 2 in glomerular endothelial cells and modulates interactions with co-cultured podocytes. Int. J. Biochem. Cell Biol. 44 1482-1490 (2012).

205. Clark, P. R. et al. MEK5 is activated by shear stress, activates ERK5 and induces KLF4 to modulate TNF responses in human dermal microvascular endothelial cells. Microcirculation 18, 102-117 (2011).

206. de Bruin, R. G. et al. The RNA-binding protein quaking maintains endothelial barrier function and affects VEcadherin and $\beta$-catenin protein expression. Sci. Rep. $\mathbf{6}$, 21643 (2016)

207. Boon, R. A. et al. KLF2-induced actin shear fibers control both alignment to flow and JNK signaling in vascular endothelium. Blood 115, 2533-2542 (2010).

208. Sangwung, P. et al. KLF2 and KLF4 control endothelial identity and vascular integrity. JCl Insight 2, e91700 (2017).

209. Frangos, J. A., Eskin, S. G., McIntire, L. V. \& Ives, C. L. Flow effects on prostacyclin production by cultured human endothelial cells. Science 227, 1477-1479 (1985). 
210. Hsieh, H. J., Liu, C. A., Huang, B., Tseng, A. H. \& Wang, D. L. Shear-induced endothelial mechanotransduction: the interplay between reactive oxygen species (ROS) and nitric oxide (NO) and the pathophysiological implications. J. Biomed. Sci. 21, 3 (2014).

211. Zhong, F. et al. Reduced Kruppel-like factor 2 expression may aggravate the endothelial injury of diabetic nephropathy. Kidney Int. 87, 382-395 (2015).

212. Zhong, F. et al. Reduced Kruppel-like factor 2 aggravates glomerular endothelial cell injury and kidney disease in mice with unilateral nephrectomy. Am. J. Pathol. 186, 2021-2031 (2016).

213. Yoshida, T., Yamashita, M., Iwai, M. \& Hayashi, M. Endothelial Kruppel-like factor 4 mediates the protective effect of statins against ischemic AKI J. Am. Soc. Nephrol. 27, 1379-1388 (2016).

214. Fan, Y. et al. Kruppel-like factors and vascular wall homeostasis. J. Mol. Cell Biol. 9, 352-363 (2017).

215. Zhou, Z. et al. Cerebral cavernous malformations arise from endothelial gain of MEKK3-KLF2/4 signalling. Nature 532, 122-126 (2016).

216. Doddaballapur, A. et al. Laminar shear stress inhibits endothelial cell metabolism via KLF2-mediated repression of PFKFB3. Arterioscler. Thromb. Vasc. Biol. 35, 137-145 (2015).

217. Chen, Z. et al. Shear stress, SIRT1, and vascular homeostasis. Proc. Natl Acad. Sci. USA 107, 10268-10273 (2010).

218. Kim, B., Lee, H., Kawata, K. \& Park, J. Y. Exercisemediated wall shear stress increases mitochondrial biogenesis in vascular endothelium. PLOS ONE 9, e111409 (2014)

219. Kim, J. S. et al. Shear stress-induced mitochondrial biogenesis decreases the release of microparticles from endothelial cells. Am. J. Physiol. Heart Circ. Physiol. 309, H425-H433 (2015).

220. Vigetti, D., Viola, M., Karousou, E., De Luca, G. \& Passi, A. Metabolic control of hyaluronan synthases. Matrix Biol. 35, 8-13 (2014).

221. Eelen, G. et al. Endothelial cell metabolism. Physiol. Rev. 98, 3-58 (2018).

222. Tammi, R. H. et al. Transcriptional and post-translational regulation of hyaluronan synthesis. FEBS J. 278, 1419-1428 (2011)

223. Wu, D. et al. HIF- 1 a is required for disturbed flowinduced metabolic reprogramming in human and porcine vascular endothelium. eLife 6, e25217 (2017).

224. Yamamoto, K., Imamura, H. \& Ando, J. Shear stress augments mitochondrial ATP generation that triggers ATP release and $\mathrm{Ca}(2+)$ signaling in vascular endothelial cells. Am. J. Physiol. Heart Circ. Physiol. 315, H1477-H1485 (2018).

225. Chen, X. L. et al. Laminar flow induction of antioxidant response element-mediated genes in endothelial cells. A novel anti-inflammatory mechanism. J. Biol. Chem. 278, 703-711 (2003).

226. Li, Y. et al. Glutaredoxin 1 mediates the protective effect of steady laminar flow on endothelial cells against oxidative stress-induced apoptosis via inhibiting Bim. Sci. Rep. 7, 15539 (2017).

227. Dupre, T. V., Jenkins, D. P., Muise-Helmericks, R. C. \& Schnellmann, R. G. The 5-hydroxytryptamine receptor $1 \mathrm{~F}$ stimulates mitochondrial biogenesis and angiogenesis in endothelial cells. Biochem. Pharmacol. 169, 113644 (2019)

228. Li, Z. et al. Kruppel-like factor 4 regulation of cholesterol-25-hydroxylase and liver $\mathrm{X}$ receptor mitigates atherosclerosis susceptibility. Circulation 136, 1315-1330 (2017).

229. Adams, C. M. et al. Cholesterol and 25-hydroxycholesterol inhibit activation of SREBPs by different mechanisms, both involving SCAP and Insigs. J. Biol. Chem. 279, 52772-52780 (2004)

230. Xiao, $\mathrm{H}$. et al. Sterol regulatory element binding protein 2 activation of NLRP3 inflammasome in endothelium mediates hemodynamic-induced atherosclerosis susceptibility. Circulation 128 , 632-642 (2013)

231. Chiu, J. J. \& Chien, S. Effects of disturbed flow on vascular endothelium: pathophysiological basis and clinical perspectives. Physiol. Rev. 91, 327-387 (2011)

232. Jiang, Y. Z. et al. Hemodynamic disturbed flow induces differential DNA methylation of endothelial Kruppellike factor 4 promoter in vitro and in vivo. Circ. Res. $115,32-43(2014)$

233. Wu, C. et al. Mechanosensitive PPAP2B regulates endothelial responses to atherorelevant hemodynamic forces. Circ. Res. 117, e41-e53 (2015).
234. Fang, Y. \& Davies, P. F. Site-specific microRNA-92a regulation of Kruppel-like factors 4 and 2 in atherosusceptible endothelium. Arterioscler. Thromb. Vasc. Biol. 32, 979-987 (2012)

235. Panchatcharam, M. et al. Mice with targeted inactivation of ppap $2 \mathrm{~b}$ in endothelial and hematopoietic cells display enhanced vascular inflammation and permeability. Arterioscler. Thromb. Vasc. Biol. 34, 837-845 (2014).

236. Lee, J. H., Kim, D., Oh, Y. S. \& Jun, H. S. Lysophosphatidic acid signaling in diabetic nephropathy. Int. J. Mol. Sci. 20, 2850 (2019).

237. Feng, S. et al. Mechanical activation of hypoxiainducible factor $1 a$ drives endothelial dysfunction at atheroprone sites. Arterioscler. Thromb. Vasc. Biol. 37, 2087-2101 (2017).

238. Yang, Y. et al. Nuclear heparanase-1 activity suppresses melanoma progression via its DNA-binding affinity. Oncogene 34, 5832-5842 (2015).

239. Eng, E. \& Ballermann, B. J. Diminished NF-кB activation and PDGF-B expression in glomerular endothelial cells subjected to chronic shear stress. Microvasc. Res. 65, 137-144 (2003)

240. Wang, L. et al. Integrin-YAP/TAZ-JNK cascade mediates atheroprotective effect of unidirectional shear flow. Nature 540, 579-582 (2016).

241. Wang, K. C. et al. Flow-dependent YAP/TAZ activities regulate endothelial phenotypes and atherosclerosis. Proc. Natl Acad. Sci. USA 113, 11525-11530 (2016)

242. Kim, J. et al. YAP/TAZ regulates sprouting angiogenesis and vascular barrier maturation. J. Clin. Invest. 127, 3441-3461 (2017)

243. Bertero, T. et al. Vascular stiffness mechanoactivates YAP/TAZ-dependent glutaminolysis to drive pulmonary hypertension. J. Clin. Invest. 126, 3313-3335 (2016).

244. Enzo, E. et al. Aerobic glycolysis tunes YAP/TAZ transcriptional activity. EMBO J. 34, 1349-1370 (2015).

245. Szeto, S. G. et al. YAP/TAZ are mechanoregulators of TCF- $\beta$-Smad signaling and renal fibrogenesis. J. Am. Soc. Nephrol. 27, 3117-3128 (2016).

246. Gwon, M. G. et al. Anti-fibrotic effects of synthetic TGF- $\beta 1$ and Smad oligodeoxynucleotide on kidney fibrosis in vivo and in vitro through inhibition of both epithelial dedifferentiation and endothelialmesenchymal transitions. FASEB J. 34, 333-349 (2020).

247. Zeng, L. et al. Sterol-responsive element-binding protein (SREBP) 2 down-regulates ATP-binding cassette transporter A1 in vascular endothelial cells: a novel role of SREBP in regulating cholesterol metabolism. J. Biol. Chem. 279, 48801-48807 (2004).

248. Dichtl, W. et al. HMG-CoA reductase inhibitors regulate inflammatory transcription factors in human endothelial and vascular smooth muscle cells. Arterioscler. Thromb. Vasc. Biol. 23, 58-63 (2003).

249. Sen-Banerjee, S. et al. Kruppel-like factor 2 as a novel mediator of statin effects in endothelial cells. Circulation 112, 720-726 (2005).

250. Sands, J. M. \& Layton, H. E. The physiology of urinary concentration: an update. Semin. Nephrol. 29, 178-195 (2009)

251. Madonna, R. et al. Simulated hyperglycemia impairs insulin signaling in endothelial cells through a hyperosmolar mechanism. Vascul. Pharmacol. 130, 106678 (2020).

252. Burg, M. B., Ferraris, J. D. \& Dmitrieva, N. I. Cellular response to hyperosmotic stresses. Physiol. Rev. 87 , 1441-1474 (2007).

253. Olsen, $H$. et al. Differential regulation of cell volume and shape in confluent rat hepatocytes under hypertonic stress. Cell Physiol. Biochem. 19, 259-268 (2007)

254. Bookstein, C. et al. A unique sodium-hydrogen exchange isoform (NHE-4) of the inner medulla of the rat kidney is induced by hyperosmolarity. J. Biol. Chem. 269, 29704-29709 (1994).

255. Hoffmann, E. K., Lambert, I. H. \& Pedersen, S. F. Physiology of cell volume regulation in vertebrates. Physiol. Rev. 89, 193-277 (2009).

256. Wang, M. Endothelium adapts to survive dehydration Nat. Rev. Nephrol. 16, 187 (2020)

257. Mohamed, T. \& Sequeira-Lopez, M. L. S. Development of the renal vasculature. Semin. Cell Dev. Biol. 91 132-146 (2019).

258. Kitamoto, Y., Tokunaga, H. \& Tomita, K. Vascular endothelial growth factor is an essential molecule for mouse kidney development: glomerulogenesis and nephrogenesis. J Clin. Invest. 99, 2351-2357 (1997).
259. Tanabe, K., Wada, J. \& Sato, Y. Targeting angiogenesis and lymphangiogenesis in kidney disease. Nat. Rev. Nephrol. 16, 289-303 (2020).

260. Reinders, M. E., Rabelink, T. J. \& Briscoe, D. M Angiogenesis and endothelial cell repair in renal disease and allograft rejection. J. Am. Soc. Nephrol. 17, 932-942 (2006)

261. Tanaka, S., Tanaka, T. \& Nangaku, M. Hypoxia as a key player in the AKI-to-CKD transition. Am. J. Physiol. Ren. Physiol. 307, F1187-F1195 (2014).

262. Yoshida, M. et al. Low-energy extracorporeal shock wave ameliorates ischemic acute kidney injury in rats. Clin. Exp. Nephrol. 23, 597-605 (2019).

263. Leonard, E. C., Friedrich, J. L. \& Basile, D. P. VEGF-121 preserves renal microvessel structure and ameliorates secondary renal disease following acute kidney injury. Am. J. Physiol. Ren. Physiol. 295, F1648-F1657 (2008).

264. Anderson, C. E. et al. The association of angiogenic factors and chronic kidney disease. BMC Nephrol. 19 117 (2018).

265. Nakagawa, T., Kosugi, T., Haneda, M., Rivard, C. J. $\&$ Long, D. A. Abnormal angiogenesis in diabetic nephropathy. Diabetes 58, 1471-1478 (2009).

266. Eelen, G., Treps, L., Li, X. \& Carmeliet, P. Basic and therapeutic aspects of angiogenesis updated. Circ. Res. 127, 310-329 (2020).

267. Potente, M., Gerhardt, H. \& Carmeliet, P. Basic and therapeutic aspects of angiogenesis. Cell 146, 873-887 (2011)

268. Rafii, S., Butler, J. M. \& Ding, B. S. Angiocrine functions of organ-specific endothelial cells. Nature 529, 316-325 (2016)

269. van den Berg, C. W. et al. Renal subcapsular transplantation of PSC-derived kidney organoids induces neo-vasculogenesis and significant glomerular and tubular maturation in vivo. Stem Cell Rep. 10, 751-765 (2018)

270. Serluca, F. C., Drummond, I. A. \& Fishman, M. C. Endothelial signaling in kidney morphogenesis: a role for hemodynamic forces. Curr. Biol. 12, 492-497 (2002).

271. Alpers, C. E., Seifert, R. A., Hudkins, K. L., Johnson, R. J. $\&$ Bowen-Pope, D. F. Developmental patterns of PDGF B-chain, PDGF-receptor, and $\alpha$-actin expression in human glomerulogenesis. Kidney Int. 42, 390-399 (1992).

272. Lindahl, P. et al. Paracrine PDGF-B/PDGF-Rbeta signaling controls mesangial cell development in kidney glomeruli. Development 125, 3313-3322 (1998).

273. Kumar, S. Cellular and molecular pathways of renal repair after acute kidney injury. Kidney Int. 93, 27-40 (2018).

274. Tasnim, F. \& Zink, D. Cross talk between primary human renal tubular cells and endothelial cells in cocultures. Am. J. Physiol. Ren. Physiol. 302, F1055-F1062 (2012).

275. Matsumoto, K. et al. Instructive role of the microenvironment in preventing renal fibrosis. Stem Cell Transl. Med. 6, 992-1005 (2017).

276. Zhang, J. et al. Endothelial lactate controls muscle regeneration from ischemia by inducing M2-like macrophage polarization. Cell Metab. 31, 1136-1153.e7 (2020).

277. Cantelmo, A. R. et al. Inhibition of the glycolytic activator PFKFB3 in endothelium induces tumor vessel normalization, impairs metastasis, and improves chemotherapy. Cancer Cell 30, 968-985 (2016).

278. Schoors, S. et al. Fatty acid carbon is essential for dNTP synthesis in endothelial cells. Nature 520 , 192-197 (2015)

279. Wong, B. W. et al. The role of fatty acid $\beta$-oxidation in lymphangiogenesis. Nature 542, 49-54 (2017).

280. Schoors, S. et al. Corrigendum: fatty acid carbon is essential for dNTP synthesis in endothelial cells. Nature 526, 144 (2015).

281. García-Caballero, M. et al. Role and therapeutic potential of dietary ketone bodies in lymph vessel growth. Nat. Metab. 1, 666-675 (2019).

282. Koning, M., van den Berg, C. W. \& Rabelink, T. J. Stem cell-derived kidney organoids: engineering the vasculature. Cell. Mol. Life Sci. 77, 2257-2273 (2020).

283. Wu, H. et al. Comparative analysis and refinement of human PSC-derived kidney organoid differentiation with single-cell transcriptomics. Cell Stem Cell 23 , 869-881.e8 (2018).

284. Subramanian, A. et al. Single cell census of human kidney organoids shows reproducibility and diminished 
off-target cells after transplantation. Nat. Commun. 10, 5462 (2019).

285. Xu, Y. et al. Endothelial PFKFB3 plays a critical role in angiogenesis. Arterioscler. Thromb. Vasc. Biol. 34 1231-1239 (2014)

286. Yu, P. et al. FGF-dependent metabolic control of vascular development. Nature 545, 224-228 (2017).

287. Vizan, P. et al. Characterization of the metabolic changes underlying growth factor angiogenic activation: identification of new potential therapeutic targets. Carcinogenesis 30, 946-952 (2009).

288. Huang, $\mathrm{H}$. et al. Role of glutamine and interlinked asparagine metabolism in vessel formation. EMBO J. 36, 2334-2352 (2017)

289. Goveia, J. et al. An integrated gene expression landscape profiling approach to identify lung tumor endothelial cell heterogeneity and angiogenic
candidates. Cancer Cell 37, 21-36.e13 (2020).

290. Glatzel, D. K. et al. Acetyl-CoA carboxylase 1 regulates endothelial cell migration by shifting the phospholipid composition. J. Lipid Res. 59, 298-311 (2018).
291. Swinnen, J. V. et al. Fatty acid synthase drives the synthesis of phospholipids partitioning into detergentresistant membrane microdomains. Biochem. Biophys. Res. Commun. 302, 898-903 (2003)

292. Fang, L. et al. Control of angiogenesis by AIBPmediated cholesterol efflux. Nature 498, 118-122 (2013).

293. Unterluggauer, H. et al. Premature senescence of human endothelial cells induced by inhibition of glutaminase. Biogerontology 9, 247-259 (2008).

Acknowledgements

S.J.D. is supported by a Marie Skłodowska-Curie individual fellowship, and M.B. by the 'Fonds voor Wetenschappelijk Onderzoek' (FWO). The work of P.C. is supported by a Federal Government Belgium grant (IUAP P7/03), long-term structural Methusalem funding by the Flemish Government, FWO the Foundation Against Cancer, a European Research Council (ERC) Advanced Research Grant (EU-ERC269073), and RegMedXB.
Author contributions

All authors prepared and revised the manuscript, and approved the final version. S.J.D, E.M. and M.B. drafted the figures.

\section{Competing interests}

The authors declare no competing interests.

\section{Peer review information}

Nature Reviews Nephrology thanks B. Ballermann, M. Goligorsky and the other, anonymous, reviewer(s) for their contribution to the peer review of this work.

\section{Publisher's note}

Springer Nature remains neutral with regard to jurisdictional claims in published maps and institutional affiliations.

\section{Supplementary information}

The online version contains supplementary material available at https://doi.org/10.1038/s41581-021-00411-9.

(c) Springer Nature Limited 2021 